\title{
Influence of the Choice of Lunar Gravity Model on Orbit Determination for Lunar Orbiters
}

\author{
Young-Rok Kim $\mathbb{D}$, Young-Joo Song, Jonghee Bae, and Bang-Yeop Kim \\ Lunar Exploration Operation Team, Korea Aerospace Research Institute, Daejeon 34133, Republic of Korea \\ Correspondence should be addressed to Young-Rok Kim; yrkim@kari.re.kr
}

Received 8 September 2017; Revised 15 December 2017; Accepted 26 December 2017; Published 1 February 2018

Academic Editor: Viktor Avrutin

Copyright (C) 2018 Young-Rok Kim et al. This is an open access article distributed under the Creative Commons Attribution License, which permits unrestricted use, distribution, and reproduction in any medium, provided the original work is properly cited.

\begin{abstract}
We examine the influence of the lunar gravity model on the orbit determination (OD) of a lunar orbiter operating in a $100 \mathrm{~km}$ high, lunar polar orbit. Doppler and sequential range measurements by three Deep Space Network antennas and one Korea Deep Space Antenna were used. For measurement simulation and OD analysis, STK11 and ODTK6 were utilized. GLGM2, LP100K, LP150Q, GRAIL420A, and GRAIL660B were used for investigation of lunar gravity model selection effect. OD results were assessed by position and velocity uncertainties with error covariance and an external orbit comparison using simulated true orbit. The effect of the lunar gravity models on the long-term OD, degree and order level, measurement-acquisition condition, and lunar altitude was investigated. For efficiency verification, computational times for the five lunar gravity models were compared. Results showed that significant improvements to OD accuracy are observed by applying a GRAIL-based model; however, applying a full order and degree gravity modeling is not always the best strategy, owing to the computational burden. Consequently, we consider that OD using GRAIL660B with $70 \times 70$ degree and order is the most efficient strategy for mission preanalysis. This study provides useful guideline for KPLO OD analysis during nominal mission operation.
\end{abstract}

\section{Introduction}

Korea's first lunar exploration program was launched in 2016. The Korea Pathfinder Lunar Orbiter (KPLO) is being developed by the Korea Aerospace Research Institute (KARI), with the nominal mission of operating in a lunar polar orbit at an altitude of $100 \mathrm{~km}$ for one year. The KPLO will carry six technical and scientific instruments: the LUnar Terrain Imager (LUTI) by KARI, wide-angle Polarimetric Camera (PolCam) by Korea Astronomy and Space Science Institute (KASI), KPLO MAGnetometer (KMAG) by Kyung Hee University (KHU), KPLO Gamma Ray Spectrometer (KGRS) by the Korea Institute of Geoscience and Mineral Resources (KIGAM), Disruption Tolerant Network experiment PayLoad (DTNPL) by the Electronics and Telecommunications Research Institute (ETRI), and the Shadow Camera (ShadowCam) by the joint NASA contribution of Arizona State University (ASU) and Malin Space Science Systems (MSSS).

For the successful operation of KPLO, development of a flight dynamics system for spacecraft navigation is essential.
In particular, orbit propagation (OP) and orbit determination (OD) are an important procedure of lunar orbiter flight dynamics operation. The orbit prediction procedure for mission planning and payload operation is associated with the OP and OD performance. Lunar gravity is a major factor regarding lunar orbiter dynamics, making the selection of a lunar gravity model critical for improved orbit accuracy and effective mission operation. For OD of KPLO mission, sequential orbit estimation using an extended Kalman filter (EKF) and a backward smoother and batch estimation using least-squares filter are considered and an accurate lunar gravity modeling has a big influence on OD performance of both filters.

Directly obtaining information from a lunar gravity model is essential for preanalysis of KPLO mission planning and operation. Various models can be applied to the lunar orbiter OD problem and while recent models have advantages, they do not always lead to productive results. The effect of different lunar gravity models on the OP performance of KPLO mission has been analyzed [1]. For successful KPLO mission navigation, analyses of lunar gravity effect 
TABLE 1: Summarized characteristics of recent lunar gravity models.

\begin{tabular}{lcc}
\hline Lunar gravity model & Mission & Max. degree and order \\
\hline GLGM2 [7] & Clementine & $70 \times 70$ \\
LP100K [8] & Lunar Prospector & $100 \times 100$ \\
LP150Q [9] & Lunar Prospector & $150 \times 150$ \\
GRAIL420A [10] & GRAIL & $420 \times 420$ \\
GRAIL660B [11] & GRAIL & $660 \times 660$ \\
\hline
\end{tabular}

on OD accuracy are also required. Previous studies have examined the effect of lunar gravity on the OD of actual lunar orbiter missions [2-6]. However, the analyses in these studies included various effects pertaining to real tracking data with noise and bias at the mission operation stage. For the mission planning and preanalysis step, a more simple and direct analysis is required.

The goal of this study is to investigate the effects of a lunar gravity model on OD and to suggest useful guidelines for KPLO mission preparation. Here, five lunar gravity models are considered: the Goddard Lunar Gravity Model (GLGM2, derived from Clementine mission [7]), having max. degree and order of 70 each; LP100K and LP150Q (derived from the Lunar Prospector mission $[8,9])$, having max. degree and order of 100 and 150, respectively; and GRAIL420A and GRAIL660B (derived from Gravity Recovery and Interior Laboratory mission $[10,11])$, having degree and order of 420 by 420 and 660 by 660 , respectively. A summary of these models is presented in Table 1. Although several GRAILbased fields such as GL900C [12], GRGM900C [13], AIUBGRK200B [14], and GrazLGM300a [15] can be utilized for the investigation, the benefits of applying more recent GRAIL models are not remarkable for the nominal lunar orbit. Therefore, we employed only the GRAIL660B model in this study to ensure a simple and clear analysis. More details regarding the history of lunar gravity models and characteristics are summarized by Song et al. [1].

Recent lunar gravity models with full degree and order offer better accuracy; however, this is not always useful. For a preliminary OD analysis of the KPLO, selection of an appropriate lunar gravity model (based on its degree and order) is needed. Section 2 discusses the settings and characteristics of measurement simulation for OD. Measurements simulation epoch, spacecraft characteristics, and ground station tracking configurations are summarized. Reference trajectory and simulated lunar orbiter orbits are also described. Settings and estimation strategies for the OD are examined in Section 3, including detailed dynamic and measurement modeling, estimated states, and the concept of lunar gravity filed modeling by a spherical harmonic series. Section 4 presents the OD results using different lunar gravity models for a long-term period, using various degree and order levels, with different measurement-acquisition conditions, and for different lunar altitudes. For efficiency verification, a computational cost analysis is also performed. Section 5 contains the conclusion and discussion.
TABLE 2: Initial orbit information of epoch for measurement simulation (1998/11/30, Moon J2000, $100 \mathrm{~km}$ altitude).

\begin{tabular}{lccc}
\hline Position & Value & Velocity & Value \\
\hline$X(\mathrm{~km})$ & -1783.4980 & $V_{x}(\mathrm{~km} / \mathrm{s})$ & -0.0040 \\
$Y(\mathrm{~km})$ & -368.9236 & $V_{y}(\mathrm{~km} / \mathrm{s})$ & -0.6623 \\
$Z(\mathrm{~km})$ & -165.2272 & $V_{z}(\mathrm{~km} / \mathrm{s})$ & 1.5020 \\
\hline
\end{tabular}

TABLE 3: Ground networks' statistics for measurement simulation.

\begin{tabular}{lccc}
\hline Measurement & Bias model & Bias sigma & White noise sigma \\
\hline Doppler & Gauss-Markov & $0.02 \mathrm{~Hz}$ & $0.003 \mathrm{~Hz}$ \\
Sequential range & Gauss-Markov & $10 \mathrm{~m}$ & $1.5 \mathrm{~m}$ \\
\hline
\end{tabular}

\section{Measurement Simulation}

For measurement simulation of lunar orbiter, information from NASA's Lunar Prospector (LP) (http://pds-geosciences .wustl.edu/lunar/prospectorcd/lp_0012/geometry/) was used to generate a true ephemeris. LP's epoch of 00:00:00 UTC, November 30, 1998, was used for our lunar orbiter measurement simulation, as shown in Table 2. The first simulated measurement was generated on 00:00:00, December 1, 1998, based on NASA's orbit ephemeris generation strategy. Note that we only used LP's ephemeris for the initial orbit information; therefore, the real LP mission orbit ephemeris does not match the simulated orbit exactly. Spacecraft parameters of the lunar orbiter were also initialized using NASA's orbit ephemeris file (e98335.dat). Based on this, the lunar orbiter dry mass was $182.47 \mathrm{~kg}$, and the spacecraft area was $2.0 \mathrm{~m}^{2}$. Drag and solar reflectivity coefficients were set to 2.0 and 1.2, respectively. NASA/JPL's Deep Space Network (DSN) antennas and the Korea Deep Space Antenna (KDSA) were utilized for the ground station configuration for the measurement simulation. DSS27 (Goldstone, USA), DSS46 (Canberra, Australia), DSS66 (Madrid, Spain), and KDSA (Geumsan, Korea) were used. In the KPLO program, the location of KDSA has not yet been determined; therefore, one of the candidates (Geumsan) was assumed. The ground network configuration is shown in Figure 1.

For measurement simulation, the spacecraft ephemeris (which is assumed as a true orbit for this study) was generated using STK11/Astrogator and information from NASA's orbit ephemeris of LP. The simulated lunar orbit has an altitude of $100 \mathrm{~km}$. The initial orbital period of the spacecraft is $117.87 \mathrm{~min}$, and the initial semimajor axis is $1838.2210 \mathrm{~km}$. Orbital period and semimajor axis at the end of period (after one month) changed to $117.80 \mathrm{~min}$ and $1837.4922 \mathrm{~km}$, respectively. Next, Doppler and sequential range measurements from four ground stations were generated by an ODTK simulator and the STK ephemeris.

In this study, the performance of KDSA was supposed to be the same as the antenna of the DSN networks. The simulated measurement statistics of the ground stations are summarized in Table 3. The Doppler bias sigma value for the Gauss-Markov model was set to $0.02 \mathrm{~Hz}$, and its white noise sigma value was $0.003 \mathrm{~Hz}$. For the sequential range, bias sigma and white noise sigma were at $10 \mathrm{~m}$ and $1.5 \mathrm{~m}$, 


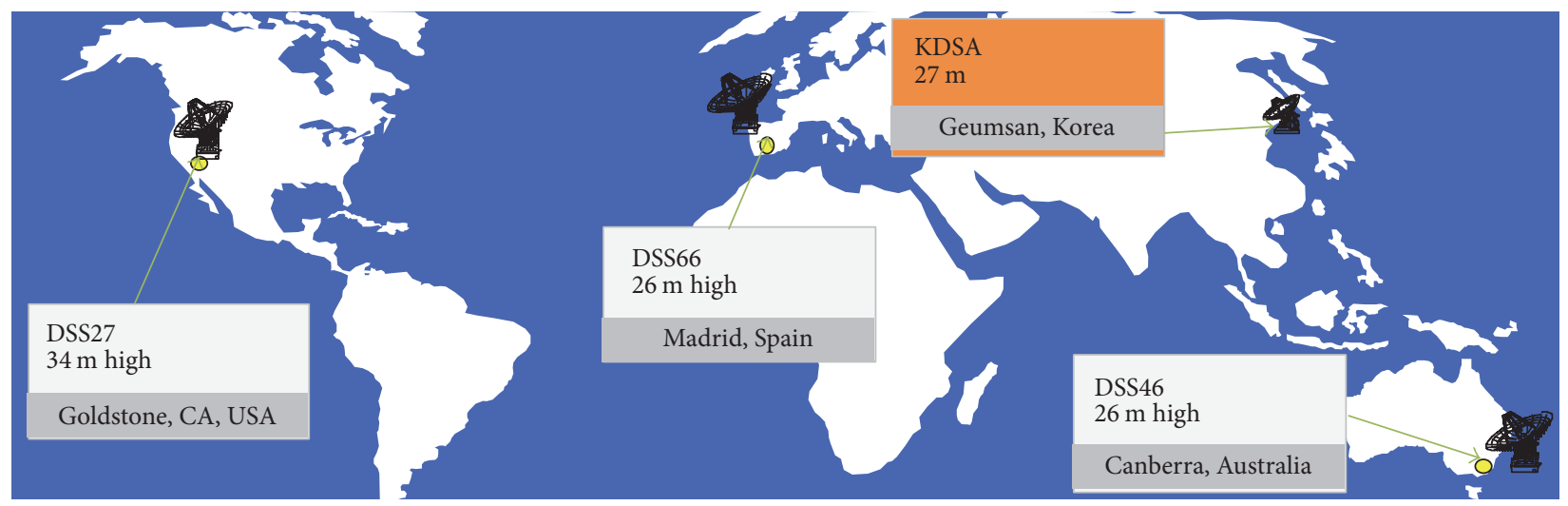

FIGURE 1: Configuration of the ground network for lunar orbiter measurement simulation and orbit determination.

respectively. The DSN service catalog for the accuracy of sequential ranging and S-band Doppler without bias were $1 \mathrm{~m}$ and $0.01 \mathrm{~mm} / \mathrm{s}$, respectively. Measurement statistics for the ground stations assume the DSN tracking performance. A total of one month of Doppler and sequential range measurements was simulated (1998/12/1-12/31). Dynamic and measurement modeling information of STK/Astrogator and ODTK simulator is as follows:

(i) Earth gravity: EGM96

(ii) Lunar gravity: GRAIL660B $660 \times 660$ (full degree and order)

(iii) Planetary ephemeris: DE430

(iv) Solid tides (full tide)

(v) General relativity correction (IERS convention)

(vi) Third body effect (Sun, Earth, and Jupiter)

(vii) Solar pressure (spherical)

(viii) Numerical integrator (RK 89, variable step size)

(ix) Troposphere and ionosphere delay correction

(x) Ground station plate motion and antenna correction

(xi) Measurement bias estimation (Gauss-Markov)

(xii) Measurement time bias estimation (Random Walk)

(xiii) Measurement simulation time step: 2 min

The mean-pass-length of each ground station was approximately $1 \mathrm{~h}$, except for the face-on geometry period. This is an ideal condition considering the fact that the orbital period of the orbiter was about $2 \mathrm{~h}$. For an investigation into the effect of lunar gravity models only, the pass length was set to theoretical values.

The total simulated measurements for a duration of one month are displayed in Figure 2. The red circle and blue triangle show simulated Doppler and sequential range measurements by four ground stations, respectively. The STK ephemeris can be considered (for OD result accuracy assessment) as a true orbit. The same model was used for an estimation of measurement bias and time bias for the OD.

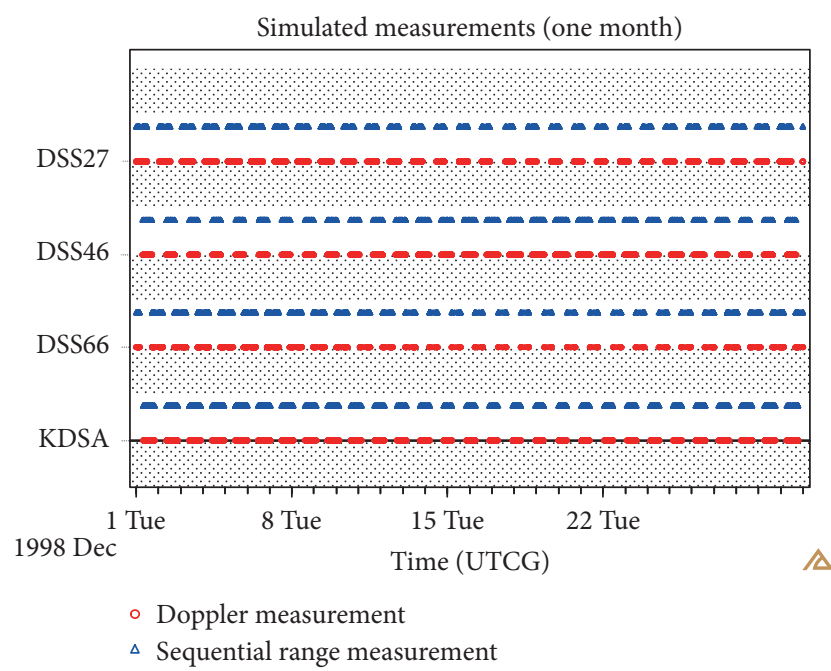

FIGURE 2: Simulated measurement for lunar orbiter (100 km altitude) orbit determination. The red circle and blue triangle are Doppler and sequential range measurements, respectively.

Because the purpose of this study is to investigate the effects of the lunar gravity model itself, the simulated measurements in this study are ideal and results cannot be directly applied to real lunar missions. Specifically, the values for accuracy analysis are not practical. For real missions, error bars would be larger than the results here.

\section{Orbit Determination}

In this section, the OD settings and estimation strategies for analysis are summarized. The OD process consists of a filter and smoother steps of the ODTK software. ODTK uses a sequential optimal filter based on the EKF. The smoother used in ODTK is a fixed interval smoother [16]. ODTK has been successfully applied to real lunar mission OD analysis, such as LRO and LADEE $[17,18]$. Because the performance of ODTK for lunar missions has already been proven, it can be assumed that the results in this OD analysis are reliable. 
TABLE 4: Adjusted initial orbit information of epoch for orbit determination (1998/12/01, Moon J2000, $100 \mathrm{~km}$ altitude).

\begin{tabular}{lccc}
\hline Position & Value & Velocity & Value \\
\hline$X(\mathrm{~km})$ & -316.7866 & $V_{x}(\mathrm{~km} / \mathrm{s})$ & 1.5694 \\
$Y(\mathrm{~km})$ & -791.6651 & $V_{y}(\mathrm{~km} / \mathrm{s})$ & 0.2124 \\
$Z(\mathrm{~km})$ & 1622.4588 & $V_{z}(\mathrm{~km} / \mathrm{s})$ & 0.4159 \\
\hline
\end{tabular}

In this study, the OD simulation consisted of five steps. First, the OD (using a one-month measurement) was performed to examine the long-term lunar gravity effect. Next, the OD using an $84 \mathrm{~h}$ measurement evaluated the specific lunar gravity effects (degree and order). Subsequently, an OD using measurement-acquisition condition was conducted. Among the various measurement-acquisition conditions, only the number of tracking stations was considered. Then, the OD for three different lunar altitudes $(50 \mathrm{~km}, 100 \mathrm{~km}$, and $200 \mathrm{~km}$ ) was performed. Finally, the computational time for each lunar gravity model was analyzed to determine the efficiency of mission operation.

The settings for the OD in this study are summarized as follows. First, the initial state of the lunar orbiter OD was set to the same epoch value as the measurement simulation $(1998 / 11 / 30)$. This means that we did not consider an initial orbit error. After the first run, a new initial epoch state $(1998 / 12 / 01)$ was updated by the smoothing process.

The final adjusted initial orbit information is shown in Table 4. Note that this process does not always improve OD characteristics or accuracy. The initial covariance values were set to $10 \mathrm{~m}$ and $0.01 \mathrm{~m} / \mathrm{s}$ for position and velocity, respectively. These values are rather small, considering the initial state adjustment. Dynamic and measurement modeling for lunar orbiter OD is as follows:

(i) Earth gravity: GGM03C

(ii) Lunar gravity: GLGM2, LP100K, LP150Q, GRAIL420A, and GRAIL660B

(iii) Planetary ephemeris: DE430

(iv) Solid tides and general relativity correction (IERS convention)

(v) Third body effect (Sun and Earth)

(vi) Solar pressure (spherical)

(vii) Troposphere and ionosphere delay correction

(viii) Ground station plate motion and antenna correction

(ix) Unmodeled accelerations (process noise) $0.1 \mathrm{~cm} / \mathrm{s}$ in $X, Y$, and $Z$ directions

(x) Measurement bias estimation (Gauss-Markov)

(xi) Measurement time bias estimation (Random Walk)

(xii) Numerical integration $(\mathrm{RK} 78$, step size $=30 \mathrm{~s})$

Estimated states are as follows:

(i) Lunar orbiter position $(X, Y$, and $Z)$

(ii) Lunar orbiter velocity $\left(V_{x}, V_{y}\right.$, and $\left.V_{z}\right)$

(iii) Solar pressure coefficient $C_{r}$ (iv) Lunar orbiter transponder bias

(v) Station measurement bias

To investigate the effects of a selected lunar gravity model, GLGM2, LP100K, LP150Q, GRAIL420A, and GRAIL660B were utilized. For the degree and order effect investigation, various values were considered. The lunar gravity field can be modeled by a spherical harmonic series as follows $[1,11]$ :

$$
\begin{gathered}
U(r, \varphi, \lambda)=\frac{G M}{r} \sum_{n=0}^{\infty} \sum_{m=0}^{n}\left(\frac{R_{e}}{r}\right)^{n} \bar{P}_{n m}(\sin \varphi) \\
\cdot\left[\bar{C}_{n m} \cos m \lambda+\bar{S}_{n m} \sin m \lambda\right],
\end{gathered}
$$

where $U$ is the lunar gravity potential; $r$ is the distance between the center of the Moon and the spacecraft; $\varphi$ and $\lambda$ are the latitude and longitude, respectively; $G$ is the universal constant of gravitation; $M$ is the mass of the Moon; $R_{e}$ is the reference equatorial radius of the Moon; $n$ and $m$ are the degree and order, respectively; $\bar{P}_{n m}$ are the fully normalized Legendre polynomials; and $\bar{C}_{n m}$ and $\bar{S}_{n m}$ are the normalized harmonic coefficients. The lunar gravity potential $(U)$ can be described in another form as follows $[1,19]$ :

$$
U(r, \varphi, \lambda)=\frac{G M}{r} \sum_{m=0}^{N}\left[A_{m}^{(0)} \cos m \lambda+B_{m}^{(0)} \sin m \lambda\right],
$$

where $A_{m}^{(0)}$ and $B_{m}^{(0)}$ are the "lumped coefficients" defined by

$$
\begin{aligned}
A_{m}^{(0)} & =\sum_{n=m}^{N}\left(\frac{R_{e}}{r}\right)^{n} \bar{C}_{n m} \bar{P}_{n m}(\varphi), \\
B_{m}^{(0)} & =\sum_{n=m}^{N}\left(\frac{R_{e}}{r}\right)^{n} \bar{S}_{n m} \bar{P}_{n m}(\varphi),
\end{aligned}
$$

where $N$ is the maximum degree value of a spherical harmonic series.

As shown in Table 1, each gravity model has a maximum degree and order value, and therefore the available numbers of degree and order for each model were limited. For example, $21 \times 21,40 \times 40,70 \times 70,100 \times 100$, and $150 \times 150$ were applied for LP150Q; and $21 \times 21,40 \times 40$, and $70 \times 70$ were employed for GLGM2. Basically, four ground stations were used for the filter and smoother process. However, for investigation of the measurement-acquisition conditions, four ground station configurations were used: KDSA only, KDSA and 1 DSN, KDSA and 2 DSNs, and KDSA and 3 DSNs. For OD performance validation, position and velocity uncertainties by using error covariance and position and velocity differences between the estimated orbit and true orbit were utilized. They demonstrate orbit precision and orbit accuracy, respectively.

\section{Results}

OD results for the effect of the lunar gravity model choice are demonstrated by orbit precision and accuracy assessment using error covariance, as well as the differences between the 
TABLE 5: Orbit propagation (OP) differences between true orbit dynamics and OD dynamics.

\begin{tabular}{lccc}
\hline & & Propagated orbit difference (m, RMS) & Cross-track (min./max.) \\
& Radial (min./max.) & In-track (min./max.) & $3.07(-7.52 / 8.56)$ \\
Position & $7.86(-14.31 / 15.36)$ & $73.53(-140.99 / 22.14)$ & $0.27(-0.70 / 0.78)$ \\
\hline
\end{tabular}

TABLE 6: Long-term lunar gravity effects on orbit determination of lunar orbiter (filter only).

\begin{tabular}{|c|c|c|c|c|c|c|c|c|}
\hline \multirow{2}{*}{ Lunar gravity model $(70 \times 70)$} & \multicolumn{4}{|c|}{ Orbit position uncertainty (m, RMS) } & \multicolumn{4}{|c|}{ Orbit position accuracy (m, RMS) } \\
\hline & $\mathrm{R}$ & I & $\mathrm{C}$ & $3 \mathrm{D}$ & $\mathrm{R}$ & I & $\mathrm{C}$ & $3 \mathrm{D}$ \\
\hline GLGM2 & 196.18 & 8443.55 & 112.33 & 8446.58 & 354426.77 & 681952.52 & 3044.51 & 768561.54 \\
\hline LP100K & 57.04 & 199.69 & 74.99 & 220.80 & 6.76 & 20.56 & 9.31 & 23.56 \\
\hline LQ150Q & 57.56 & 201.46 & 75.73 & 222.79 & 6.80 & 21.03 & 9.32 & 23.99 \\
\hline GRAIL420A & 20.01 & 52.83 & 16.26 & 58.79 & 3.24 & 10.06 & 3.58 & 11.16 \\
\hline GRAIL660B & 20.01 & 52.83 & 16.26 & 58.79 & 3.24 & 10.07 & 3.58 & 11.16 \\
\hline
\end{tabular}

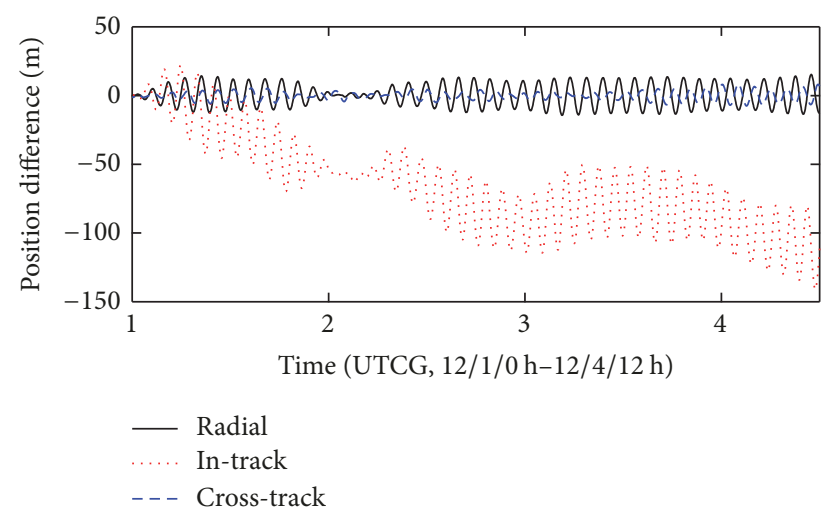

FIGURE 3: Position differences between true orbit dynamics and OD dynamics.

true orbit and determined orbit. For efficiency verification, the computational time for each OD process was also considered. Before the main analysis, the differences between the dynamics for true orbit generation (STK) and those for OD (ODTK) were investigated. In this work, the same propagation algorithm (STK and ODTK use the same orbit propagator) was used for the true orbit generation and OD. For more realistic OD simulation and analysis, intentional differences were introduced between the true orbit dynamics and OD dynamics by using different modeling parameters. To investigate the dynamic modeling differences, OP results from STK and ODTK (using the same initial state, epoch, and duration) were compared.

Table 5 lists the OP differences between the dynamics of true orbit generation and those of OD in an $84 \mathrm{~h}$ duration. The RMS values for the position differences between the true orbit dynamics and OD dynamics are $7.86 \mathrm{~m}$ for radial, $73.53 \mathrm{~m}$ for in-track (along-track), and $3.07 \mathrm{~m}$ for cross-track directions. Figures 3 and 4 show the position and velocity differences for the period between true orbit dynamics and OD dynamics, respectively. It was seen that the maximum position difference was reached at about $150 \mathrm{~m}$ in the intrack direction. The maximum velocity difference is about

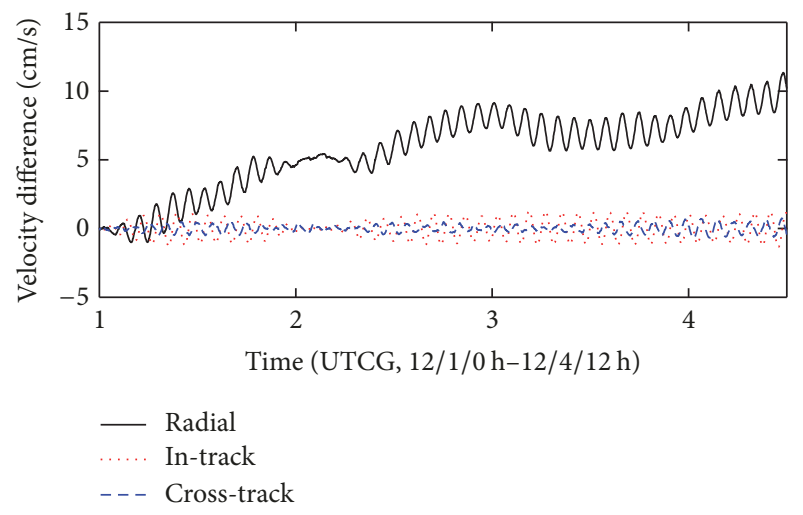

FIGURE 4: Velocity differences between true orbit dynamics and OD dynamics.

$12 \mathrm{~cm} / \mathrm{s}$ in the radial direction. Minimum and maximum value differences are also provided in Table 5.

4.1. Long-Term Lunar Gravity Effect on OD. In this subsection, the OD results for a one-month timeframe using five lunar gravity models (GLGM2, LP100K, LP150Q, GRAIL420A, and GRAIL660B) are presented. To investigate the effects of a long-term lunar gravity model, we analyzed mainly the position/velocity uncertainty using error covariance and orbit position/velocity accuracy by true orbit. The degree and order of the lunar gravity model was limited to $70 \times 70$, because GLGM2 has a maximum degree and order of 70. In this case, a sequential filter was run for the duration of one month, and the smoother was run backward. For actual mission operation, OD during a long-term period (such as a month) is not a useful approach. However, in the mission planning and preanalysis step, an OD analysis for the long-term period provides critical information such as the orbit performance trend on Earth and the lunar orbit geometry (face-on/edge-on views).

The RMS values of orbit position uncertainty and accuracy for radial, in-track, and cross-track (RIC) directions are listed in Table 6 . These values are the results of the filter 

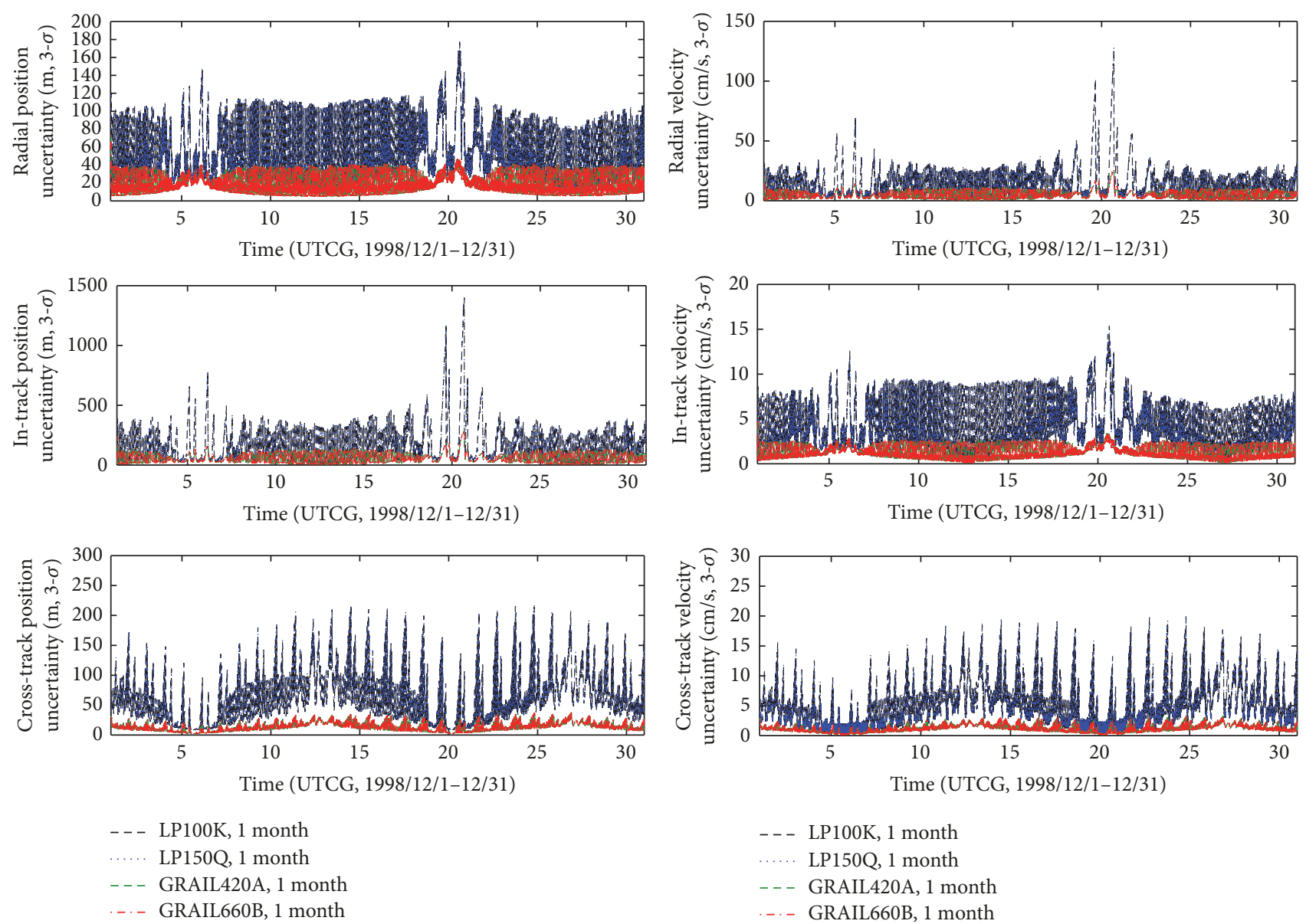

FIGURE 5: Position and velocity uncertainties based on the covariance of OD (filter only).

because GLGM2 cannot deliver reliable smoother results due to a few accepted measurements. In contrast to the other four models, GLGM2 has an unacceptable orbit error for the one-month period. The orbit uncertainties and accuracies of LP100K and LP150Q appear to be similar, and GRAIL420A and GRAIL660B indicate equivalent performance, which is also the best among the others.

Figure 5 shows the position and velocity uncertainties for RIC based on error covariance. The results of GLGM2 are excluded because they describe the divergence filter performance for one month. LP100K (black) and LP150Q (blue) exhibit similar performance, and GRAIL420A (green) and GRAIL660B (red) demonstrate smaller position and velocity uncertainty. Radial and in-track directions deliver poor uncertainty in the face-on geometry (around 12/5 and $12 / 20$ ). For the cross-track direction, the face-on geometry period presents a good uncertainty value. Face-on geometry means that the orbit plane and line of sight from the Earth are perpendicular; therefore, radial and in-track directions have poor observability due to small variations of sequential range and Doppler values. On the other hand, the cross-track direction has good observability at the face-on view, and orbit uncertainty demonstrates the best accuracy.

Figure 6 shows the orbit position and velocity accuracies (as calculated by differences between the determined orbit and the true orbit) for the four gravity models (LP100K, LP150Q, GRAIL420A, and GRAIL660B). The GLGM2 model is also excluded in Figure 6, because the OD using GLGM2 during a one-month period has a large error and is not acceptable.

Figure 7 shows the measurement residuals of OD by GRAIL660B. Sequential range residuals and Doppler residuals indicate that the OD for one month is successfully achieved. The RMS values of sequential range residuals and Doppler residuals are $2.36 \mathrm{~m}$ and $0.013 \mathrm{~Hz}$, respectively. It was noted that the RMS values of measurement residuals have smaller values than those of orbit uncertainty and accuracy for GRAIL660B, as shown in Table 6. The measurement residual is an indicator of the internal consistency of the OD process; therefore, orbit uncertainty by error covariance and orbit accuracy by true orbit are also analyzed to evaluate the OD performance. Occasionally, a few accepted measurement residuals lead to small measurement residuals and error covariance. Thus, considering both orbit uncertainty and orbit difference is essential for OD performance evaluation.

4.2. Degree and Order of Lunar Gravity Effect on OD. In this subsection, the OD results for an $84 \mathrm{~h}$ period using the five lunar gravity models are presented. The filter is run for an $84 \mathrm{~h}$ period and the smoother is run backward 

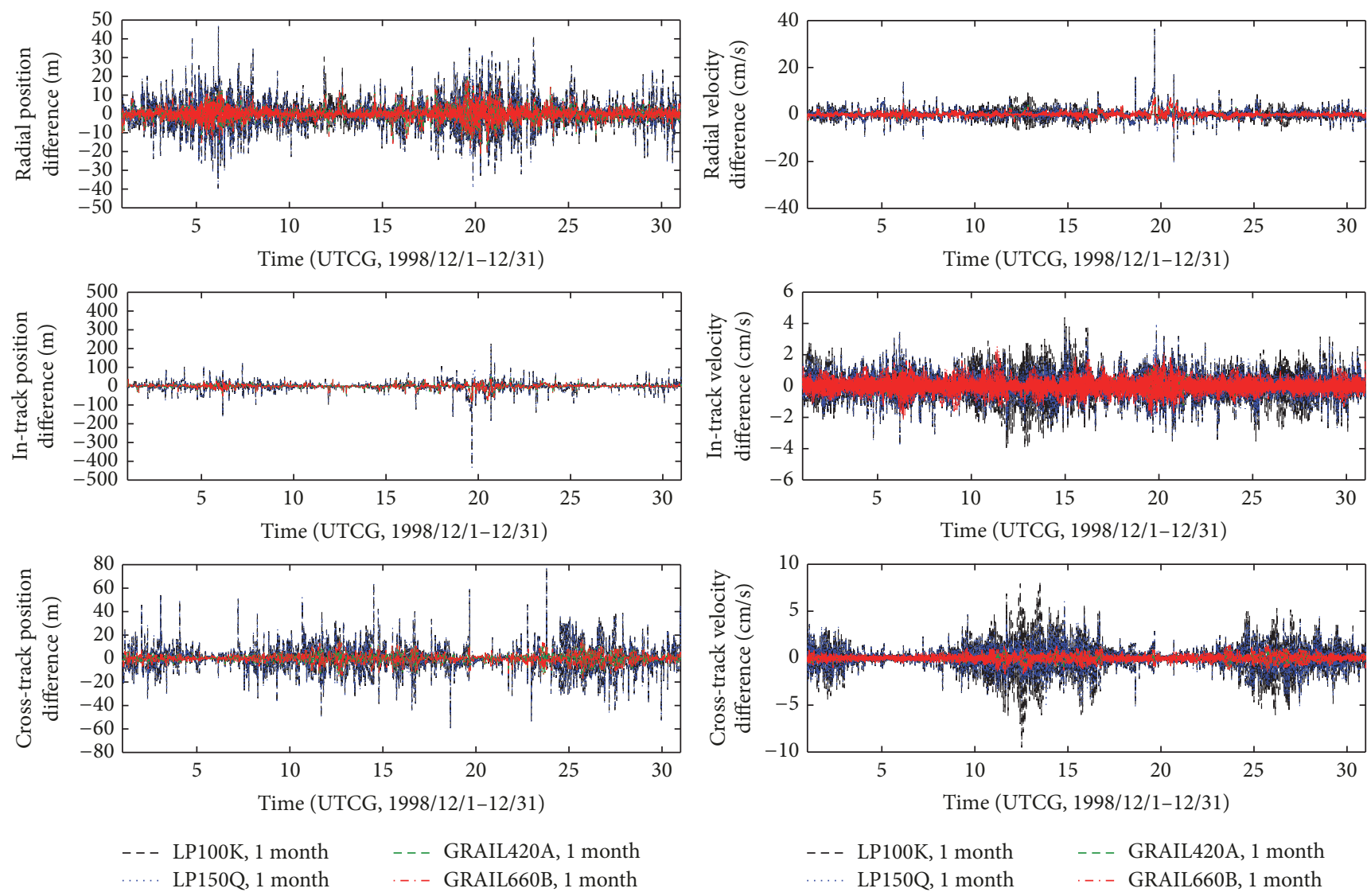

FIGURE 6: Position and velocity differences between estimated orbit and true orbit (filter only).
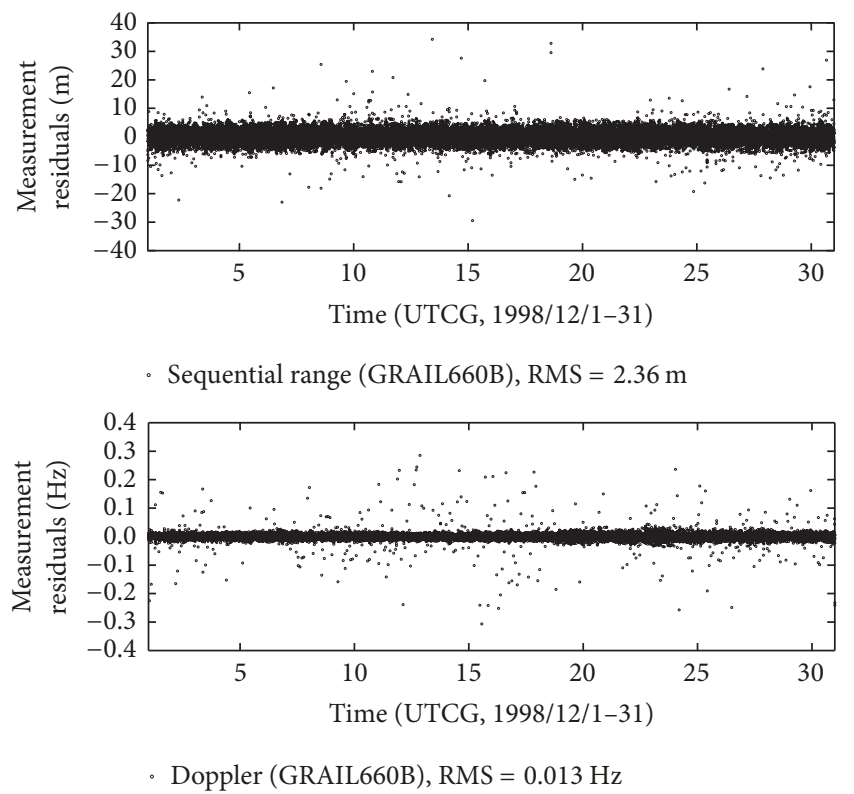

Figure 7: Measurement residuals of lunar orbiter OD using GRAIL660B model.

for the same duration. For investigation of the degree and order effect of the lunar gravity models, various degree and order values were applied. The analysis here is done mainly by position/velocity uncertainty and orbit accuracy by position/velocity difference between the estimated orbit and true orbit.

Table 7 summarizes the RMS values for position uncertainties and accuracies for three degree and order options (21 $\times 21,40 \times 40$, and $70 \times 70$ ), based on the GLGM2 gravity model. All the three degree and order combinations for the GLGM2 demonstrate bad orbit precision and accuracy for $84 \mathrm{~h}$. It can be clearly seen that the radial and cross-track directions show similar position uncertainty and accuracy regardless of the degree and order level. It is observed that the $70 \times 70$ degree and order option delivers the best orbit precision and accuracy, considering $3 \mathrm{D}$ total values for the $84 \mathrm{~h}$ track. Large position uncertainty and accuracy using GLGM2 result from the frequent rejections of large measurement residuals during sequential orbit estimation.

Table 8 presents the RMS values of position uncertainties and accuracies of four degree and order levels using the LP100K gravity model $(21 \times 21,40 \times 40,70 \times 70$, and $100 \times$ $100)$. It is clearly shown that orbit position uncertainty and accuracy are dramatically improved as compared to those in the GLGM2 results. The $21 \times 21$ option delivers the largest orbit uncertainty and accuracy in all directions. Moreover, the $100 \times 100$ option demonstrates the best OD performance. In addition, OD using LP100K for degree and order greater than $70 \times 70$ delivers a similar orbit precision and accuracy 
TABLE 7: Influence of degree and order of GLGM2 model on orbit determination of lunar orbiter.

\begin{tabular}{|c|c|c|c|c|c|c|c|c|}
\hline \multirow{2}{*}{ Lunar gravity model (GLGM2) } & \multicolumn{4}{|c|}{ Orbit position uncertainty (m, RMS) } & \multicolumn{4}{|c|}{ Orbit position accuracy (m, RMS) } \\
\hline & $\mathrm{R}$ & I & $\mathrm{C}$ & $3 \mathrm{D}$ & $\mathrm{R}$ & I & C & $3 \mathrm{D}$ \\
\hline $21 \times 21$ & 57.95 & 344.75 & 63.17 & 355.25 & 1094.67 & 16841.59 & 2531.78 & 17065.97 \\
\hline $40 \times 40$ & 58.59 & 306.53 & 55.83 & 317.04 & 1108.16 & 13931.97 & 2497.67 & 14197.40 \\
\hline $70 \times 70$ & 64.33 & 244.71 & 61.15 & 260.31 & 1150.14 & 9116.97 & 2508.43 & 9525.45 \\
\hline
\end{tabular}

TABLE 8: Influence of degree and order of LP100K model on orbit determination of lunar orbiter.

\begin{tabular}{lccccccc}
\hline $\begin{array}{l}\text { Lunar gravity model } \\
\text { (LP100K) }\end{array}$ & \multicolumn{3}{c}{ Orbit position uncertainty (m, RMS) } & \multicolumn{3}{c}{ Orbit position accuracy (m, RMS) } \\
\hline $21 \times 21$ & $\mathrm{R}$ & $\mathrm{I}$ & $\mathrm{C}$ & 3D & R & I & C \\
$40 \times 40$ & 35.00 & 94.26 & 60.07 & 117.12 & 8.29 & 21.53 & 13.72 \\
$70 \times 70$ & 22.20 & 60.17 & 38.17 & 74.64 & 4.31 & 11.97 & 6.43 \\
$100 \times 100$ & 21.47 & 58.23 & 36.92 & 72.21 & 3.31 & 9.18 & 5.33 \\
\hline
\end{tabular}

Table 9 lists the RMS values of position uncertainties and accuracies obtained using various degree and order combinations with the LP150Q gravity model $(21 \times 21,40 \times$ $40,70 \times 70,100 \times 100$, and $150 \times 150)$. It is clear that the same trend within LP100K is observed. As shown in Table 9, degree and order options over $70 \times 70$ give similar orbit precision and accuracy for the RIC and 3D. It is also seen that the orbit precision and accuracy of LP100K and LP150Q do not have large differences.

Tables 10 and 11 list the RMS values of the position uncertainties and accuracies obtained using seven degree and order combinations for GRAIL420A $(21 \times 21,40 \times 40,70$ $\times 70,100 \times 100,200 \times 200,300 \times 300$, and $420 \times 420)$ and GRAIL660B $(21 \times 21,40 \times 40,70 \times 70,100 \times 100$, $200 \times 200,300 \times 300$, and $660 \times 660$ ), respectively. It is clear that the GRAIL-based gravity models deliver the best orbit precision and accuracy among the five considered lunar gravity models. GRAIL420A and GRAIL660B demonstrate equivalent performance in terms of position uncertainties and accuracies. Figure 8 shows the differences between the orbit position accuracies of GRAIL420A and GRAIL660B. It is seen that the accuracy differences between the two models do not exceed $1 \mathrm{~m}$ for RIC directions. This indicates that GRAIL420A and GRAIL660B exhibit the same OD performance. For both models, OD accuracy improvement is observed when using the $70 \times 70$ degree and order combination.

Figures 9 and 10 show the position/velocity uncertainties and accuracies corresponding to various degree and order levels for the GRAIL660B model. For GRAIL660B, as shown in Figures 9 and 10, an OD with degree and order greater than $70 \times 70$ guarantees stable orbit precision and accuracy. Additionally, it is seen that the uncertainty patterns (due to measurement gap) are removed using degree and order greater than $70 \times 70$.

4.3. Measurement-Acquisition Condition and Lunar Gravity Effect on $O D$. In this subsection, $O D$ results for an $84 \mathrm{~h}$ period using five lunar gravity models and various measurement-acquisition conditions are presented. For
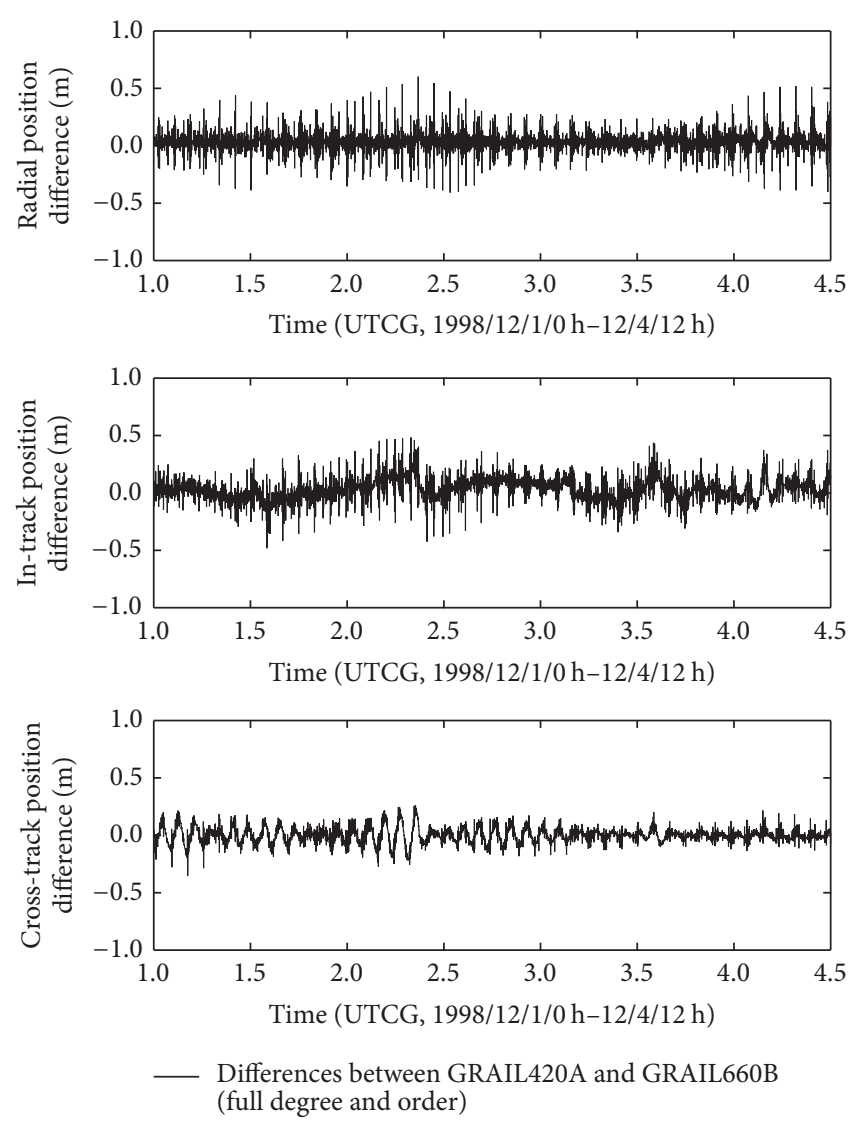

FIGURE 8: Differences in position accuracy between GRAIL420A and GRAIL660B.

investigation of measurement-acquisition condition, four ground station configurations (KDSA only, KDSA and DSS66, KDSA and DSS66/27, and KDSA and DSS66/27/46) were applied. Each lunar gravity model used a $70 \times 70$ degree and order coefficient.

Tables 12-15 summarize the effects of measurementacquisition conditions on lunar orbiter position uncertainty 

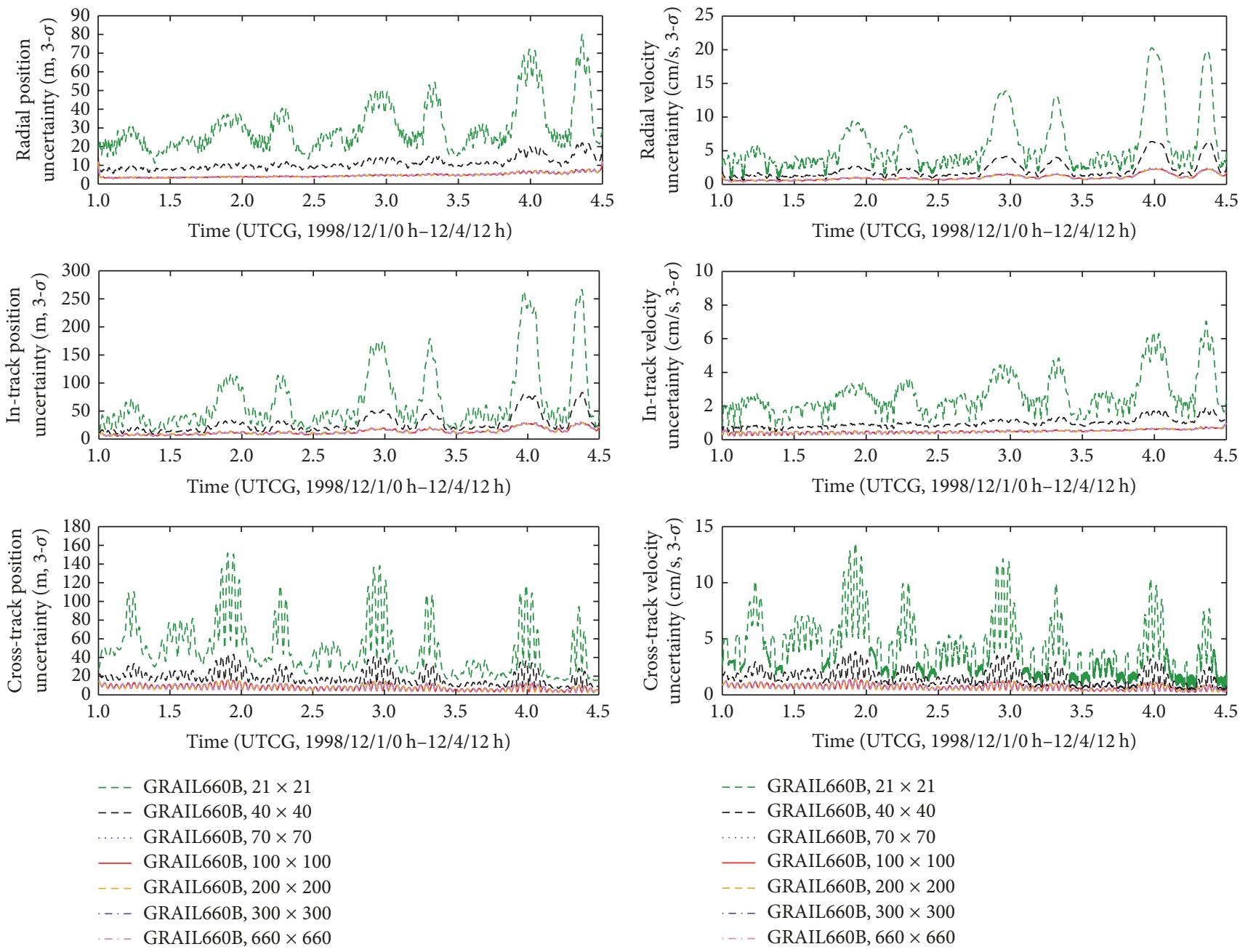

FIGURE 9: Position and velocity uncertainties based on the covariance of OD (GRAIL660B).

TABLE 9: Influence of degree and order of LP150Q model on orbit determination of lunar orbiter.

\begin{tabular}{lcccccrrr}
\hline $\begin{array}{l}\text { Lunar gravity model } \\
(\mathrm{LP150Q})\end{array}$ & \multicolumn{3}{c}{ Orbit position uncertainty $(\mathrm{m}, \mathrm{RMS})$} & \multicolumn{3}{c}{ Orbit position accuracy (m, RMS) } \\
\hline $21 \times 21$ & $\mathrm{R}$ & $\mathrm{I}$ & $\mathrm{C}$ & 3D & $\mathrm{R}$ & $\mathrm{I}$ & $\mathrm{C}$ & 3D \\
$40 \times 40$ & 33.70 & 90.78 & 57.87 & 112.81 & 8.27 & 20.82 & 13.81 \\
$70 \times 70$ & 22.38 & 60.66 & 38.49 & 75.24 & 4.27 & 12.21 & 6.66 & 26.32 \\
$100 \times 100$ & 21.68 & 58.79 & 37.28 & 72.91 & 3.29 & 9.26 & 5.36 & 14.55 \\
$150 \times 150$ & 21.56 & 58.49 & 37.08 & 72.53 & 3.27 & 9.24 & 5.35 \\
\hline
\end{tabular}

TABLE 10: Influence of degree and order of GRAIL420A model on orbit determination of lunar orbiter.

\begin{tabular}{lccccccc}
\hline $\begin{array}{l}\text { Lunar gravity model } \\
\text { (GRAIL420A) }\end{array}$ & \multicolumn{3}{c}{ Orbit position uncertainty (m, RMS) } & \multicolumn{3}{c}{ Orbit position accuracy (m, RMS) } \\
\hline $21 \times 21$ & $\mathrm{R}$ & $\mathrm{I}$ & $\mathrm{C}$ & 3D & $\mathrm{R}$ & $\mathrm{I}$ & $\mathrm{C}$ \\
$40 \times 40$ & 32.73 & 88.27 & 56.26 & 109.67 & 7.97 & 19.12 & 13.20 \\
$70 \times 70$ & 11.45 & 31.81 & 19.50 & 39.02 & 3.46 & 10.16 & 5.79 \\
$100 \times 100$ & 4.99 & 14.94 & 8.71 & 18.00 & 0.86 & 2.88 & 1.61 \\
$200 \times 200$ & 4.73 & 14.26 & 8.30 & 17.17 & 0.61 & 2.10 & 1.24 \\
$300 \times 300$ & 4.72 & 14.25 & 8.29 & 17.15 & 0.62 & 2.08 & 1.23 \\
$420 \times 420$ & 4.72 & 14.25 & 8.29 & 17.15 & 0.62 & 2.08 & 1.22 \\
\hline
\end{tabular}



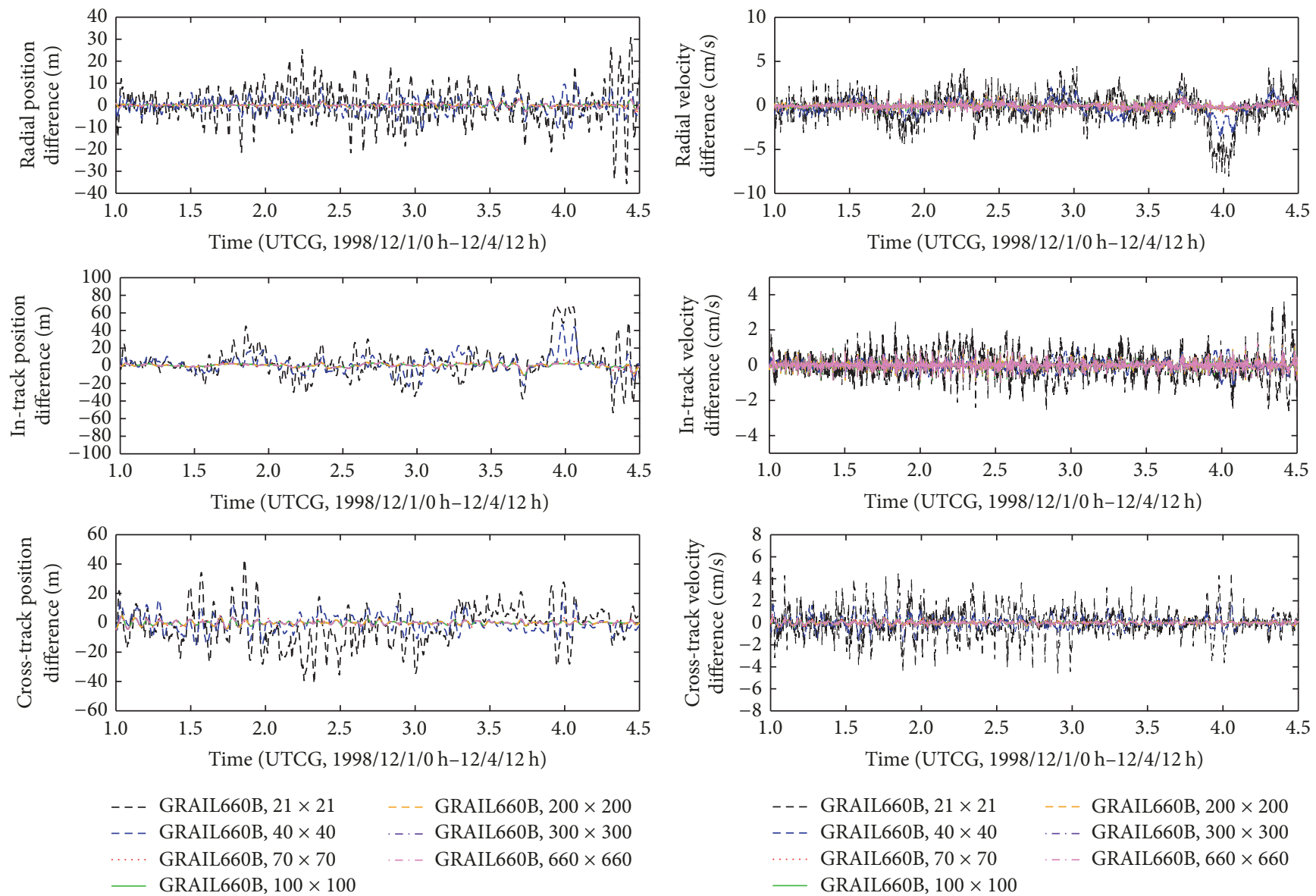

FIGURE 10: Position and velocity differences between estimated orbit and true orbit (GRAIL660B).

TABLE 11: Influence of degree and order of GRAIL660B model on orbit determination of lunar orbiter.

\begin{tabular}{lccccccc}
\hline $\begin{array}{l}\text { Lunar gravity model } \\
\text { (GRAIL660B) }\end{array}$ & \multicolumn{3}{c}{ Orbit position uncertainty (m, RMS) } & \multicolumn{4}{c}{ Orbit position accuracy (m, RMS) } \\
\hline $21 \times 21$ & $\mathrm{R}$ & $\mathrm{I}$ & $\mathrm{C}$ & 3D & $\mathrm{R}$ & $\mathrm{I}$ & C \\
$40 \times 40$ & 32.74 & 88.27 & 56.26 & 109.68 & 8.04 & 19.12 & 13.14 \\
$70 \times 70$ & 11.45 & 31.81 & 19.50 & 39.02 & 3.46 & 10.18 & 5.80 \\
$100 \times 100$ & 4.99 & 14.94 & 8.71 & 18.00 & 0.86 & 2.89 & 1.63 \\
$200 \times 200$ & 4.73 & 14.26 & 8.30 & 17.17 & 0.62 & 2.12 & 1.24 \\
$300 \times 300$ & 4.72 & 14.25 & 8.29 & 17.15 & 0.62 & 2.09 & 1.25 \\
$660 \times 660$ & 4.72 & 14.25 & 8.29 & 17.15 & 0.63 & 2.13 & 1.26 \\
\hline
\end{tabular}

TABLE 12: Influence of lunar gravity model on orbit determination of lunar orbiter by one station.

\begin{tabular}{|c|c|c|c|c|c|c|c|c|}
\hline \multirow{2}{*}{ Lunar gravity model $(70 \times 70)$} & \multicolumn{4}{|c|}{ Orbit position uncertainty (m, RMS) } & \multicolumn{4}{|c|}{ Orbit position accuracy (m, RMS) } \\
\hline & $\mathrm{R}$ & I & $\mathrm{C}$ & $3 \mathrm{D}$ & $\mathrm{R}$ & I & C & $3 \mathrm{D}$ \\
\hline GLGM2 & 94.38 & 358.89 & 66.92 & 377.08 & 1104.88 & 5534.25 & 2538.68 & 6188.18 \\
\hline LP100K & 89.98 & 882.03 & 342.08 & 950.31 & 3.57 & 22.09 & 8.23 & 23.84 \\
\hline LQ150Q & 91.02 & 892.27 & 346.37 & 961.46 & 3.63 & 22.42 & 8.51 & 24.26 \\
\hline GRAIL420A & 29.88 & 253.44 & 50.48 & 260.14 & 2.77 & 18.84 & 2.05 & 19.16 \\
\hline GRAIL660B & 29.88 & 253.44 & 50.48 & 260.14 & 2.77 & 18.86 & 2.02 & 19.16 \\
\hline
\end{tabular}


TABLE 13: Influence of lunar gravity model on orbit determination of lunar orbiter by two stations.

\begin{tabular}{|c|c|c|c|c|c|c|c|c|}
\hline \multirow{2}{*}{ Lunar gravity model $(70 \times 70)$} & \multicolumn{4}{|c|}{ Orbit position uncertainty (m, RMS) } & \multicolumn{4}{|c|}{ Orbit position accuracy (m, RMS) } \\
\hline & $\mathrm{R}$ & I & C & $3 \mathrm{D}$ & $\mathrm{R}$ & I & C & $3 \mathrm{D}$ \\
\hline GLGM2 & 64.21 & 250.28 & 64.26 & 266.26 & 1039.76 & 8111.28 & 2530.49 & 8560.21 \\
\hline LP100K & 38.58 & 248.83 & 104.71 & 272.71 & 2.35 & 18.31 & 7.02 & 19.75 \\
\hline LQ150Q & 39.03 & 251.79 & 105.98 & 275.95 & 2.34 & 17.55 & 6.80 & 18.97 \\
\hline GRAIL420A & 9.96 & 46.38 & 16.74 & 50.31 & 1.17 & 5.23 & 2.29 & 5.83 \\
\hline GRAIL660B & 9.96 & 46.38 & 16.74 & 50.31 & 1.17 & 5.26 & 2.34 & 5.87 \\
\hline
\end{tabular}

TABLE 14: Influence of lunar gravity model on orbit determination of lunar orbiter by three stations.

\begin{tabular}{|c|c|c|c|c|c|c|c|c|}
\hline \multirow{2}{*}{ Lunar gravity model $(70 \times 70)$} & \multicolumn{4}{|c|}{ Orbit position uncertainty (m, RMS) } & \multicolumn{4}{|c|}{ Orbit position accuracy (m, RMS) } \\
\hline & $\mathrm{R}$ & I & $\mathrm{C}$ & $3 \mathrm{D}$ & $\mathrm{R}$ & I & $\mathrm{C}$ & $3 \mathrm{D}$ \\
\hline GLGM2 & 53.90 & 297.48 & 56.04 & 307.48 & 1202.32 & 14203.89 & 2511.31 & 14474.21 \\
\hline LP100K & 23.42 & 67.20 & 43.04 & 83.17 & 2.89 & 9.38 & 5.11 & 11.07 \\
\hline LQ150Q & 23.66 & 67.88 & 43.49 & 84.02 & 2.90 & 9.56 & 5.16 & 11.25 \\
\hline GRAIL420A & 5.11 & 16.20 & 9.60 & 19.51 & 0.67 & 2.36 & 1.60 & 2.93 \\
\hline GRAIL660B & 5.11 & 16.20 & 9.60 & 19.51 & 0.67 & 2.35 & 1.61 & 2.93 \\
\hline
\end{tabular}

TABLE 15: Influence of lunar gravity model on orbit determination of lunar orbiter by four stations.

\begin{tabular}{|c|c|c|c|c|c|c|c|c|}
\hline \multirow{2}{*}{ Lunar gravity model $(70 \times 70)$} & \multicolumn{4}{|c|}{ Orbit position uncertainty (m, RMS) } & \multicolumn{4}{|c|}{ Orbit position accuracy (m, RMS) } \\
\hline & $\mathrm{R}$ & I & C & $3 \mathrm{D}$ & $\mathrm{R}$ & I & $\mathrm{C}$ & $3 \mathrm{D}$ \\
\hline GLGM2 & 64.33 & 244.71 & 61.15 & 260.31 & 1150.14 & 9116.97 & 2508.43 & 9525.45 \\
\hline LP100K & 21.47 & 58.23 & 36.92 & 72.21 & 3.31 & 9.18 & 5.33 & 11.12 \\
\hline LQ150Q & 21.68 & 58.79 & 37.28 & 72.91 & 3.29 & 9.26 & 5.36 & 11.19 \\
\hline GRAIL420A & 4.99 & 14.94 & 8.71 & 18.00 & 0.86 & 2.88 & 1.61 & 3.41 \\
\hline GRAIL660B & 4.99 & 14.94 & 8.71 & 18.00 & 0.86 & 2.89 & 1.63 & 3.43 \\
\hline
\end{tabular}

and accuracy. Orbit position accuracy results for four ground station configurations show that GLGM2 exhibits a remarkably worse performance than the other gravity models. GLGM2 with KDSA only and KDSA and DSS66 configurations deliver better position uncertainty than LP100K and LP150Q because the OD precision by error covariance is a result of accepted measurements during the sequential OD process. Actual OD performance using GLGM2 must be confirmed by orbit position accuracy results. Orbit position differences show that OD precision of the GLGM2 model is not the actual precision. Therefore, OD analysis using only error covariance can lead to an unreliable conclusion. Figure 11 demonstrates the effect of lunar gravity model on lunar orbiter position uncertainty and accuracy for four ground station configurations. It is clearly seen that GRAILbased gravity models (GRAIL420A and GRAIL660B) exhibit the best OD performance, and position uncertainty and accuracy are improved by adding stations. LP100K and LP150Q have similar differences for all ground station configurations. It was also seen that the uncertainty and accuracy differences between OD by three stations and that by four stations are not significant; this is because DSS46 and KDSA are located in similar ground conditions, as shown in Figure 1.

Figure 12 shows the position and velocity uncertainties of OD results using the GRAIL660B lunar gravity models and four ground station configurations. For comparisons by ground station configuration, uncertainties and accuracies are presented at the same scale. The orbit precision and accuracy are improved by adding ground stations for measurement acquisition. The large uncertainty during some periods, as shown in Figure 12, was owing to a lack of tracking data as a result of too few ground stations. These gaps can be reduced by an increase in ground stations. The measurement gap is thus another unstable characteristic in lunar orbiter OD. Figure 13 shows position and velocity differences for four ground station configurations. Radial position accuracy appears to be less affected (relatively) by station number; however, the in-track and cross-track position accuracies are improved with an increase in number of ground stations. For velocity accuracy, in-track accuracy is less affected by station number, and other direction accuracies are improved by an increased number of stations.

4.4. Effect of Lunar Altitude and Lunar Gravity Model on OD. This subsection presents the OD results for three different altitudes during an $84 \mathrm{~h}$ period. This paper focuses on an altitude of $100 \mathrm{~km}$ for KPLO mission operation. However, it is necessary to analyze OD performance above and below $100 \mathrm{~km}$ for the prenominal and extended mission or contingency situations. To investigate the effect of lunar 

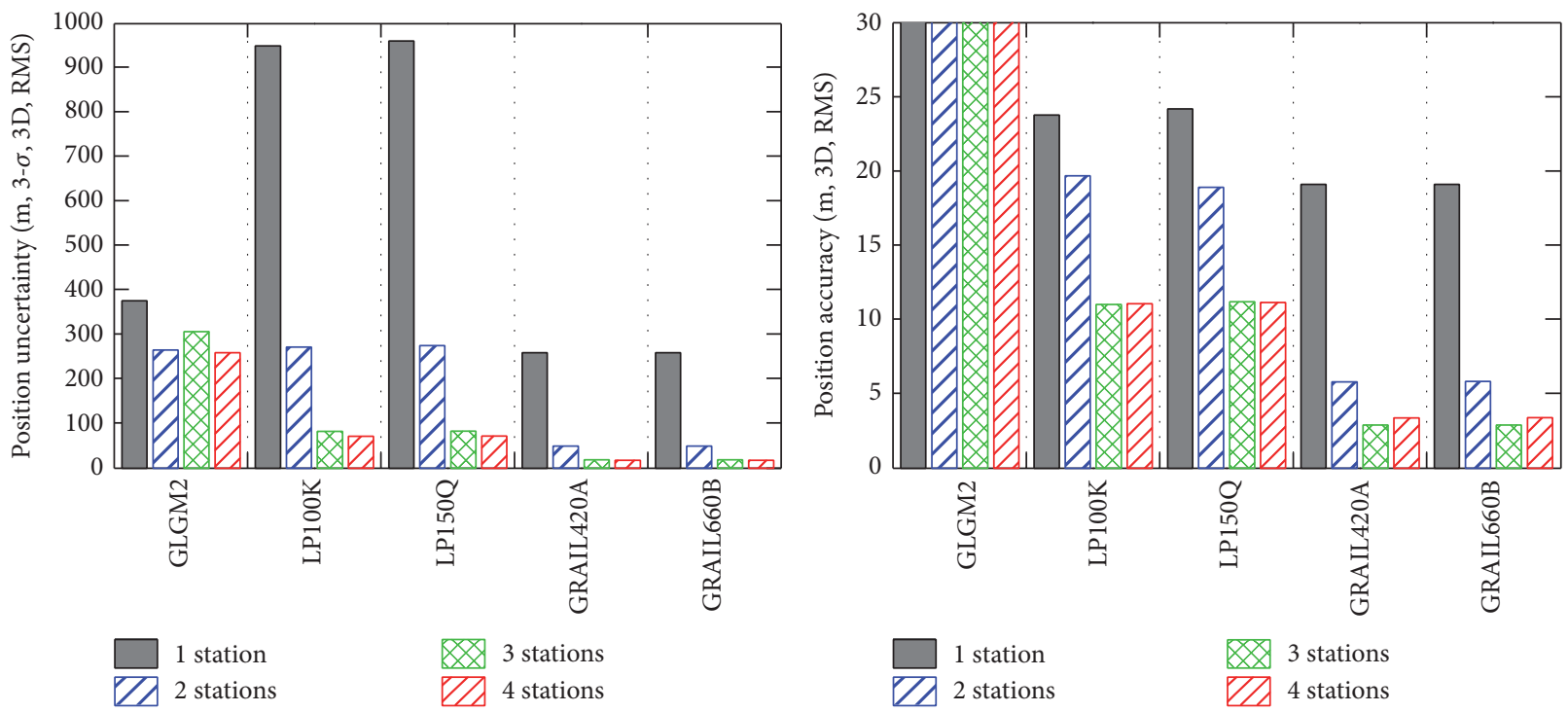

FIGURE 11: Influence of lunar gravity model on orbit position uncertainty and accuracy using four ground station configurations.
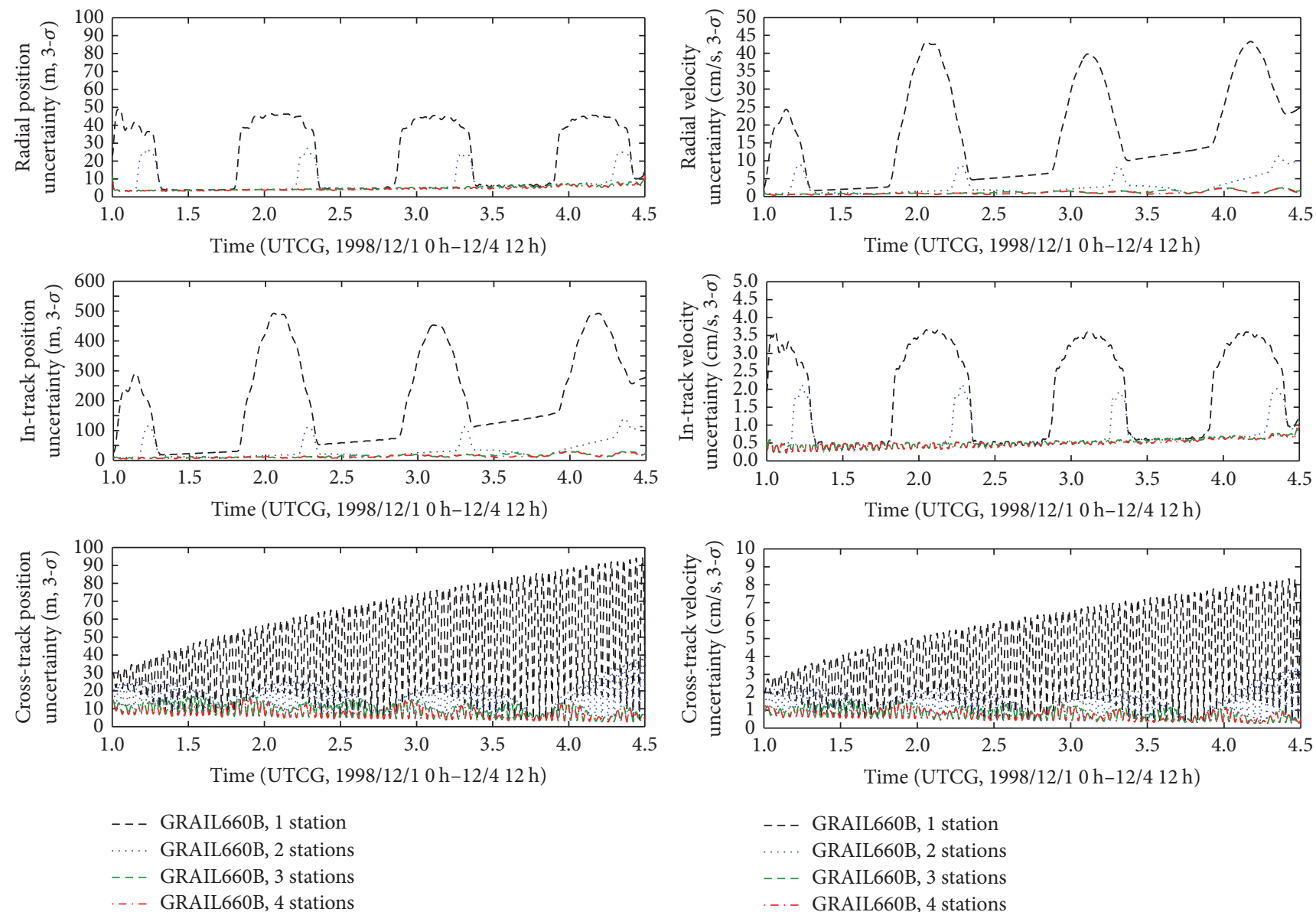

- - - GRAIL660B, 1 station
.... GRAIL660B, 2 stations
- - - GRAIL660B, 3 stations
..- GRAIL660B, 4 stations

FIgURE 12: Position and velocity uncertainties based on the covariance of OD for four ground station configurations. 

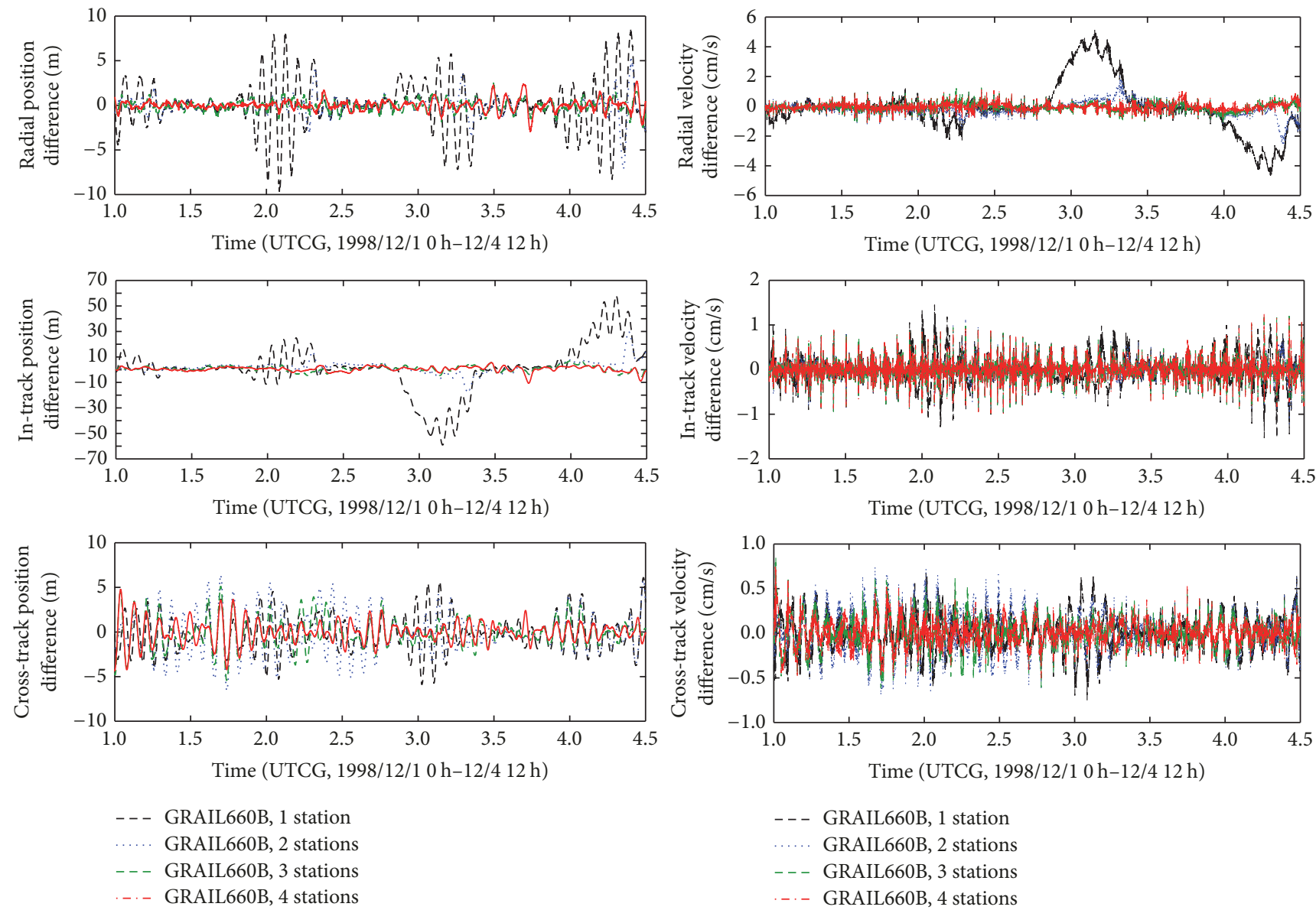

FIGURE 13: Position and velocity differences between estimated orbit and true orbit for four ground station configurations.

TABLE 16: Effect of lunar altitude of lunar gravity model on orbit determination of lunar orbiter.

\begin{tabular}{|c|c|c|c|c|c|c|c|c|c|}
\hline \multirow{2}{*}{$\begin{array}{l}\text { Lunar gravity } \\
\text { model }\end{array}$} & \multirow{2}{*}{$\begin{array}{l}\text { Lunar } \\
\text { altitude }\end{array}$} & \multicolumn{4}{|c|}{ Orbit position uncertainty (m, RMS) } & \multicolumn{4}{|c|}{ Orbit position accuracy (m, RMS) } \\
\hline & & $\mathrm{R}$ & I & $\mathrm{C}$ & $3 \mathrm{D}$ & $\mathrm{R}$ & I & $\mathrm{C}$ & $3 \mathrm{D}$ \\
\hline \multirow{3}{*}{$\begin{array}{l}\text { GLGM2 } \\
(70 \times 70)\end{array}$} & $50 \mathrm{~km}$ & 66.37 & 461.50 & 59.46 & 470.03 & 1118.06 & 40373.16 & 4528.51 & 40641.73 \\
\hline & $100 \mathrm{~km}$ & 64.33 & 244.71 & 61.15 & 260.31 & 1150.14 & 9116.97 & 2508.43 & 9525.45 \\
\hline & $200 \mathrm{~km}$ & 56.53 & 322.47 & 59.11 & 332.68 & 685.62 & 6611.22 & 794.69 & 6694.01 \\
\hline \multirow{3}{*}{$\begin{array}{l}\text { LP100K } \\
(100 \times 100)\end{array}$} & $50 \mathrm{~km}$ & 37.63 & 102.75 & 67.89 & 128.78 & 5.79 & 17.85 & 12.43 & 22.51 \\
\hline & $100 \mathrm{~km}$ & 21.33 & 57.88 & 36.69 & 71.77 & 3.26 & 9.16 & 5.31 & 11.08 \\
\hline & $200 \mathrm{~km}$ & 10.58 & 28.18 & 17.43 & 34.78 & 1.77 & 4.73 & 2.70 & 5.72 \\
\hline \multirow{3}{*}{$\begin{array}{l}\text { LQ150Q } \\
(150 \times 150)\end{array}$} & $50 \mathrm{~km}$ & 37.80 & 103.16 & 68.18 & 129.30 & 6.34 & 19.79 & 13.62 & 24.85 \\
\hline & $100 \mathrm{~km}$ & 21.49 & 58.29 & 36.96 & 72.29 & 3.27 & 9.23 & 5.33 & 11.15 \\
\hline & $200 \mathrm{~km}$ & 10.72 & 28.53 & 17.66 & 35.23 & 1.76 & 4.72 & 2.69 & 5.71 \\
\hline \multirow{3}{*}{$\begin{array}{l}\text { GRAIL420A } \\
(420 \times 420)\end{array}$} & $50 \mathrm{~km}$ & 4.66 & 14.38 & 8.32 & 17.26 & 0.92 & 3.76 & 2.15 & 4.43 \\
\hline & $100 \mathrm{~km}$ & 4.72 & 14.25 & 8.29 & 17.15 & 0.62 & 2.08 & 1.23 & 2.50 \\
\hline & $200 \mathrm{~km}$ & 5.04 & 14.65 & 8.69 & 17.76 & 0.69 & 2.79 & 1.80 & 3.39 \\
\hline \multirow{3}{*}{$\begin{array}{l}\text { GRAIL660B } \\
(660 \times 660)\end{array}$} & $50 \mathrm{~km}$ & 4.66 & 14.38 & 8.32 & 17.26 & 0.91 & 3.81 & 2.16 & 4.47 \\
\hline & $100 \mathrm{~km}$ & 4.72 & 14.25 & 8.29 & 17.15 & 0.62 & 2.09 & 1.25 & 2.51 \\
\hline & $200 \mathrm{~km}$ & 5.04 & 14.65 & 8.69 & 17.76 & 0.70 & 2.78 & 1.79 & 3.38 \\
\hline
\end{tabular}

altitude of the lunar gravity models on OD, we additionally simulated true orbits and tracking measurements for a lunar orbiter located at altitudes of 50 and $200 \mathrm{~km}$, using the same methodology with different initial orbit values as presented in Section 2. The maximum degree and order values of each lunar gravity model were applied to minimize performance degradation due to degree and order truncation.

Table 16 summarizes the RMS values for position uncertainty and accuracy for three different altitudes $(50 \mathrm{~km}$, $100 \mathrm{~km}$, and $200 \mathrm{~km}$ ) in each lunar gravity model with 
TABLE 17: Computational times for lunar orbiter orbit determination using various gravity models.

\begin{tabular}{|c|c|c|c|c|c|}
\hline \multirow{2}{*}{ Lunar gravity model } & \multicolumn{2}{|c|}{$\begin{array}{l}\text { Long term }(70 \times 70 \text {, } \\
\text { one month })\end{array}$} & \multicolumn{3}{|c|}{ Degree and order $(84 \mathrm{~h})$} \\
\hline & Total & (Filter + smoother $)$ & & Total & $\begin{array}{c}\text { (Filter }+ \\
\text { smoother) }\end{array}$ \\
\hline \multirow{3}{*}{ GLGM2 } & \multirow{3}{*}{$409 \mathrm{~s}$} & \multirow{3}{*}{$(394 s+15 s)$} & $21 \times 21$ & $19 \mathrm{~s}$ & $(16 s+3 s)$ \\
\hline & & & $40 \times 40$ & $27 s$ & $(24 s+3 s)$ \\
\hline & & & $70 \times 70$ & $50 \mathrm{~s}$ & $(43 s+7 s)$ \\
\hline \multirow{4}{*}{ LP100K } & \multirow{4}{*}{$448 \mathrm{~s}$} & \multirow{4}{*}{$(392 s+56 s)$} & $21 \times 21$ & $19 \mathrm{~s}$ & $(16 s+3 s)$ \\
\hline & & & $40 \times 40$ & $26 s$ & $(23 s+3 s)$ \\
\hline & & & $70 \times 70$ & $55 s$ & $(47 s+8 s)$ \\
\hline & & & $100 \times 100$ & $86 s$ & $(75 s+11 s)$ \\
\hline \multirow{5}{*}{ LP150Q } & \multirow{5}{*}{$442 \mathrm{~s}$} & \multirow{5}{*}{$(386 s+56 s)$} & $21 \times 21$ & $19 \mathrm{~s}$ & $(16 s+3 s)$ \\
\hline & & & $40 \times 40$ & $27 s$ & $(23 s+4 s)$ \\
\hline & & & $70 \times 70$ & $54 \mathrm{~s}$ & $(48 s+6 s)$ \\
\hline & & & $100 \times 100$ & $85 \mathrm{~s}$ & $(74 s+11 s)$ \\
\hline & & & $150 \times 150$ & $168 \mathrm{~s}$ & $(148 s+20 s)$ \\
\hline \multirow{7}{*}{ GRAIL420A } & \multirow{7}{*}{$424 \mathrm{~s}$} & \multirow{7}{*}{$(375 s+49 s)$} & $21 \times 21$ & $17 \mathrm{~s}$ & $(15 s+2 s)$ \\
\hline & & & $40 \times 40$ & $27 \mathrm{~s}$ & $(24 s+3 s)$ \\
\hline & & & $70 \times 70$ & $49 s$ & $(43 s+6 s)$ \\
\hline & & & $100 \times 100$ & $80 \mathrm{~s}$ & $(70 s+10 s)$ \\
\hline & & & $200 \times 200$ & $270 s$ & $(237 s+33 s)$ \\
\hline & & & $300 \times 300$ & $720 s$ & $(627 s+75 s)$ \\
\hline & & & $420 \times 420$ & $1669 \mathrm{~s}$ & $(1502 s+167 s)$ \\
\hline \multirow{7}{*}{ GRAIL660B } & \multirow{7}{*}{$430 \mathrm{~s}$} & \multirow{7}{*}{$(377 s+53 s)$} & $21 \times 21$ & $18 \mathrm{~s}$ & $(16 s+2 s)$ \\
\hline & & & $40 \times 40$ & $28 \mathrm{~s}$ & $(24 s+4 s)$ \\
\hline & & & $70 \times 70$ & $51 \mathrm{~s}$ & $(44 s+7 s)$ \\
\hline & & & $100 \times 100$ & $86 \mathrm{~s}$ & $(75 s+11 s)$ \\
\hline & & & $200 \times 200$ & $285 \mathrm{~s}$ & $(250 s+35 s)$ \\
\hline & & & $300 \times 300$ & $662 \mathrm{~s}$ & $(583 s+79 s)$ \\
\hline & & & $660 \times 660$ & $7314 \mathrm{~s}$ & $(6679 s+635 s)$ \\
\hline
\end{tabular}

maximum degree and order. The lunar orbiter located at $50 \mathrm{~km}$ exhibits the largest position uncertainty and accuracy for GLGM2, LP100K, and LP150Q. As shown in Table 16, for pre-GRAIL models (GLGM2, LP100K, and LP150Q), we can expect that OD for lunar orbiter at a higher altitude delivers better precision and accuracy. This means that lunar altitude is a major influencing factor in OD using pre-GRAIL models. On the other hand, GRAIL420A and GRAIL660B show similar OD performance for all three altitudes, and the best performance in terms of position uncertainty and accuracy is noted for the $100 \mathrm{~km}$ altitude; this indicates that altitude is not a critical factor in OD using GRAIL-based models. It is proven that GRAIL-based models demonstrate the best position uncertainty and accuracy for lunar orbiter OD. Figure 14 shows the effect of lunar altitude on the position uncertainty and accuracy obtained using four lunar gravity models. GRAIL420A and GRAIL660B demonstrate stable and accurate OD performance against different lunar altitudes. Figure 15 shows detailed trends of position differences between estimated orbit and true orbit for three altitudes using GRAIL660B. Although all three cases show similar OD position accuracies, each case has a different precision pattern. The variations in position accuracy are less than $15 \mathrm{~m}$; therefore, it can be regarded as stable orbit accuracy for mission operation.
4.5. Efficiency Verification by Computational Time Analysis. In this subsection, the computational times for OD, using five lunar gravity models, are investigated for efficiency verification. Table 17 lists the computational times for a onemonth and $84 \mathrm{~h}$ lunar orbiter OD, using the five lunar gravity models. For practical considerations, the computational time for an OD is divided into the filtering and smoothing time.

For a long-term OD (using the measurements of one month), GLGM2 had the shortest computational time. Its filtering time had a similar value to that of LP100K; however, the smoothing time was much shorter than that of other models. This is because the OD results using GLGM2 for one month are based on a few accepted measurements; therefore the smoothing was not performed properly. The most efficient OD results for one month were derived by GRAIL420A, which had a seven-minute processing time.

For the $84 \mathrm{~h} \mathrm{OD}$, using various degree and order levels, it is clear that the OD results with high degrees and orders lead to greater computational burdens. For the same degree and order, each lunar gravity model had the same computational times. This means that the computational time for an OD based on the sequential filter is not affected by the selection of a lunar gravity model. GRAIL660B, with full degree and order $(660 \times 660)$, required a computational capacity of more than a hundred times that of the $70 \times 70$. From an operational 

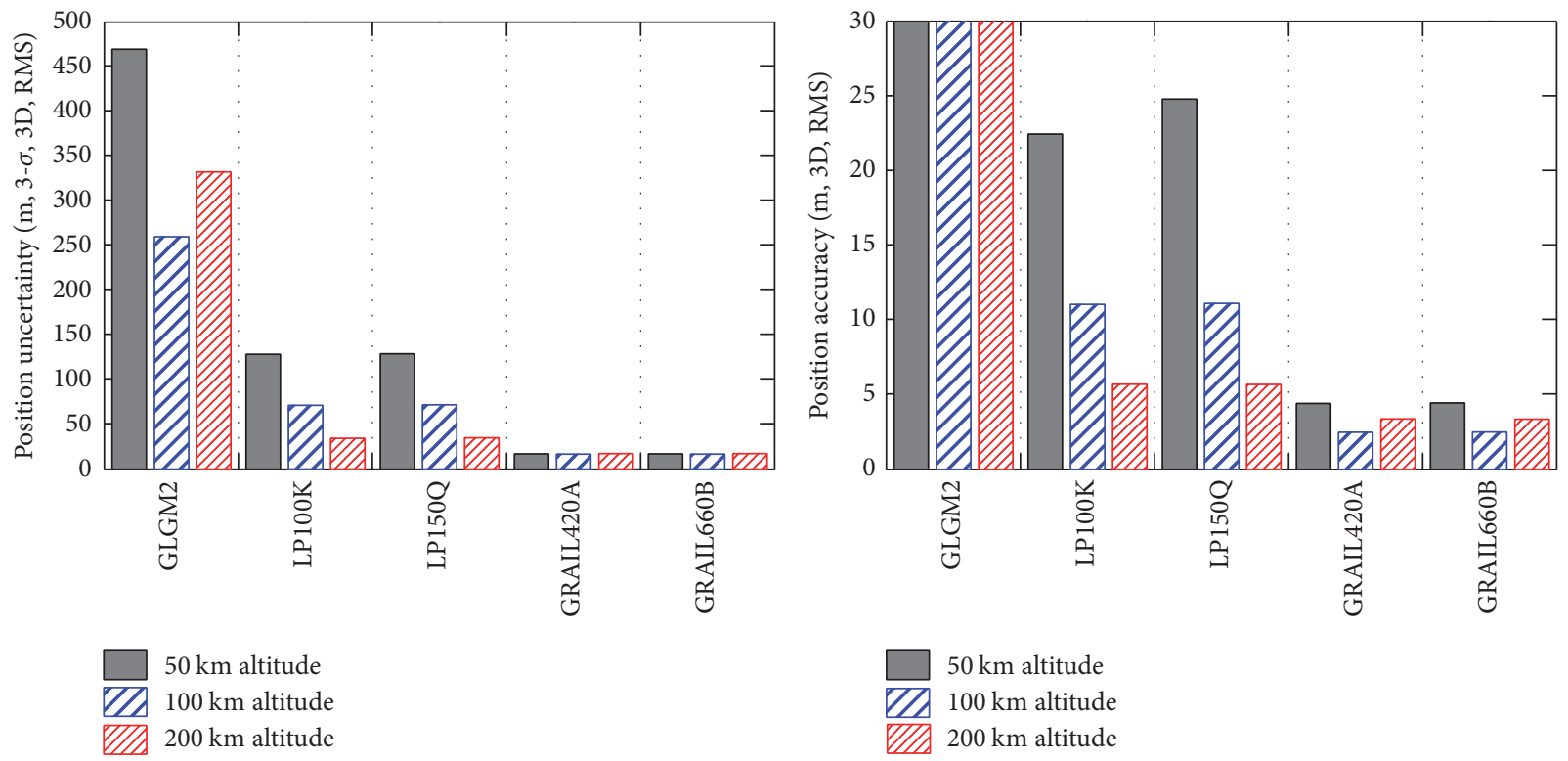

$50 \mathrm{~km}$ altitude

VI $100 \mathrm{~km}$ altitude

VIA $200 \mathrm{~km}$ altitude

$200 \mathrm{~km}$ altitude

FIGURE 14: Effect of lunar altitude on orbit position and accuracy corresponding to three altitudes.
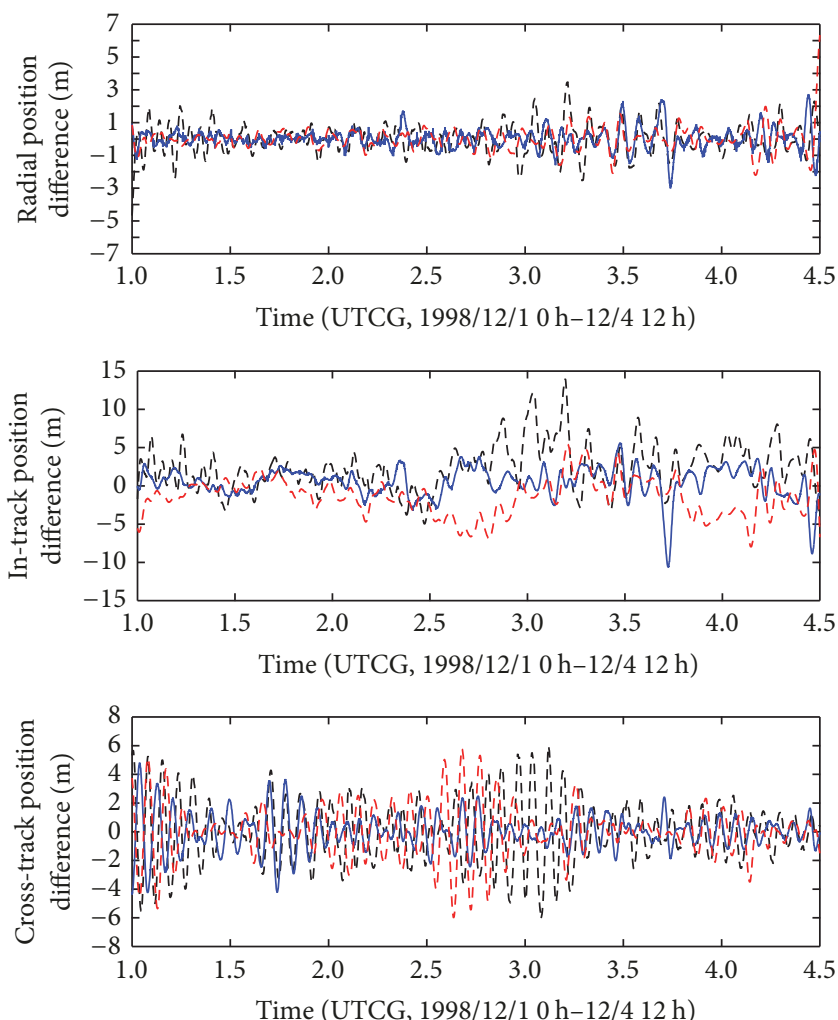

- - - GRAIL660B, $50 \mathrm{~km}$

— GRAIL660B, $100 \mathrm{~km}$

- - - GRAIL660B, $200 \mathrm{~km}$

FIGURE 15: Position differences between estimated orbit and true orbit for three lunar altitudes (GRAIL660B). 


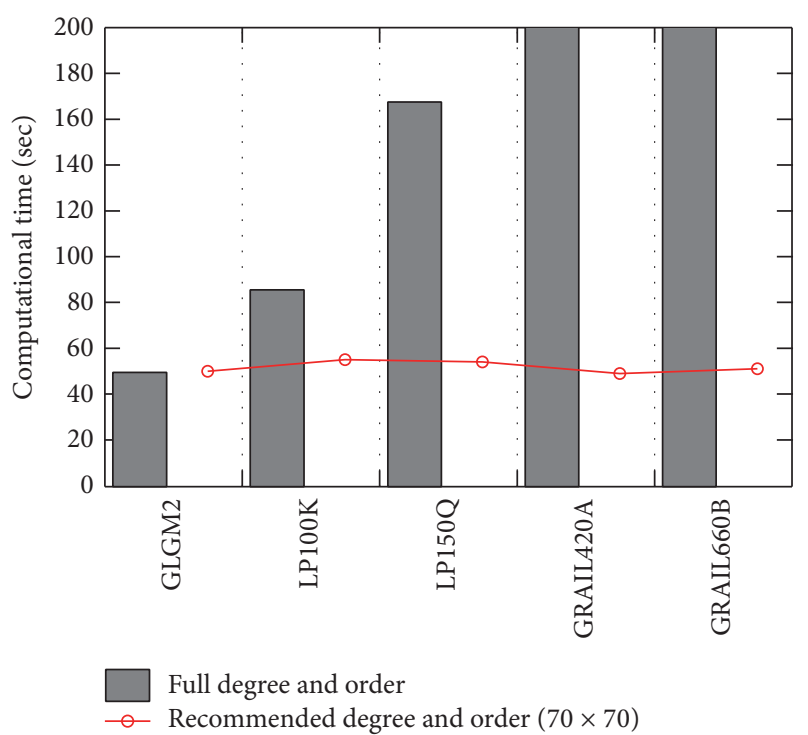

Figure 16: Computational times for full and recommended degree and order $(70 \times 70)$.

viewpoint, a full degree and order using GRAIL660B is not adequate for mission operations.

Considering the computational cost versus OD performance, we conclude that a $70 \times 70$ degree and order is the most effective choice for mission operation and analysis. As shown in Table 17, the computation times for LP100K and LP150Q are longer than that of GRAIL660B. As a matter of fact, the filtering and smoothing times are related to OD performance, seeing that the results by GRAIL660B for one month show a shorter computational burden than LP100K and LP150Q. However, this difference is not critical because (in real mission operation) the OD is accomplished using a shorter period than one month. For example, KPLO mission considers a $24 \mathrm{~h}$ and $8 \mathrm{~h}$ OD schedule for lunar and transfer orbits, respectively. In this situation, the computational time is not a critical factor for mission operation efficiency. However, during abnormal conditions or contingency situations, tuning of the operational OD can be repeated until the requirements are fulfilled. Therefore, a more efficient lunar gravity model configuration for OD must be considered for mission operations. Figure 16 shows the computational times for full degree and order and $70 \times 70$ degree and order of each lunar gravity model. It is clearly shown that the recommended $70 \times 70$ degree and order options have similar computational time for all lunar gravity models. It was also discovered that OD with GRAIL-based models with full degree and order is not an effective strategy for mission operation.

\section{Conclusions}

This paper analyzed the effect of lunar gravity model choice on orbit determination (OD) for a lunar orbiter at a $100 \mathrm{~km}$ high, lunar polar orbit. For consideration of Korea Pathfinder Lunar Orbiter (KPLO) mission, three antennas from the Deep Space Network (DSN) and the Korea Deep Space Antenna (KDSA) were selected for measurement acquisition.
To investigate the effects of a long-term lunar gravity model on OD, examinations were completed using five lunar gravity models (GLGM2, LP100K, LP150Q, GRAIL420A, and GRAIL660B), in a one-month timeframe, with a $70 \times$ 70 degree and order. To examine the effects of the degree and order level, OD results for an $84 \mathrm{~h}$ timeframe using various degree and orders were collected. Investigations into measurement-acquisition conditions were conducted using OD results for an $84 \mathrm{~h}$ timeframe using four-station acquisition configurations (KDSA only, KDSA and DSS66, KDSA and DSS66/27, and KDSA and DSS66/27/46). Additionally, OD results for lunar orbiter located at different lunar altitudes $(50 \mathrm{~km}, 100 \mathrm{~km}$, and $200 \mathrm{~km})$ for an $84 \mathrm{~h}$ timeframe were analyzed for the prenominal and extended mission or contingency situations.

From the long-term OD analysis (using the one-month measurements), GLGM2 exhibits inadequate performance, and GRAIL-based models (GRAIL420A and GRAIL660B) demonstrate equivalent and the best orbit accuracy. From the investigation of degree and order effect, using an $84 \mathrm{~h}$ timeframe, OD using a greater than $70 \times 70$ degree and order level has similar performance. From the analysis of measurementacquisition conditions effect, GRAIL-based models show the best accuracy for all ground station conditions and maintain a relatively stable accuracy when using only a few ground stations. OD analysis for three different lunar altitudes shows that OD accuracy decreases for the lunar orbiter at lower altitude using pre-GRAIL models (GLGM2, LP100K, and LP150Q). However, OD using GRAIL-based models demonstrates constant orbit precision and accuracy regardless of lunar altitude. This indicates that lunar altitude is not a critical influencing factor for OD accuracy in GRAIL-based models. Note that the values for orbit precision and accuracy in this work are based on theoretical assumptions to simplify the OD for a lunar orbiter. Therefore, it is unreasonable to directly apply the obtained OD performance data to real missions.

The efficiency verification of a computational time analysis demonstrated that a high degree and order level carries intensive computational costs, and the selection of a lunar gravity model does not affect the computational burden of sequential OD using the same degree and order levels. As a result, GRAIL-based models with a $70 \times 70$ degree and order deliver the optimal OD performance for both accuracy and efficiency. After analysis, we recommend that KPLO mission operations use a GRAIL660B model with a $70 \times 70$ degree and order as the best strategy. Although GRAIL420A and GRAIL660B have the same performance, 660 full degree and order options can be more advantageous.

It is expected that recent lunar gravity models with high degree and order capabilities deliver better OD precision and accuracy. From a technical view, it is necessary to examine how OD performance improves, or degrades, with various lunar gravity models and their degree and order options. As seen from the results of Section 4, OD precision and accuracy are affected by the selection of a lunar gravity model and its degree and order level. For a long-term OD simulation, the selection of the lunar gravity model, and its degree and order, is a critical factor due to the computational time. 
The OD results presented in this paper provide a practical guideline for the estimation of the total analysis time for OD. In this study, a $100 \mathrm{~km}$ altitude was used for KPLO mission, and altitudes of 50 and $200 \mathrm{~km}$ were additionally considered. If the mission altitude decreases during an extended mission, or due to contingency situations, the effect on the lunar gravity models and degree and order becomes much greater. In these cases, a more efficient and accurate OD analysis is essential for stable mission operations.

\section{Conflicts of Interest}

The authors declare that there are no conflicts of interest regarding the publication of this paper.

\section{Acknowledgments}

This work was supported by the Korea Aerospace Research Institute (KARI) (under a contract with the Ministry of Science and ICT) through the "Development of Pathfinder Lunar Orbiter and Key Technologies for the Second Stage Lunar Exploration" project (no. SR17026).

\section{References}

[1] Y.-J. Song, Y.-R. Kim, J. Bae, and B.-Y. Kim, "Evolution of the Selenopotential Model and Its Effects on the Propagation Accuracy of Orbits around the Moon," Mathematical Problems in Engineering, vol. 2017, Article ID 5493679, 2017.

[2] S. E. Slojkowski, "Lunar Reconnaissance Orbiter orbit determination accuracy analysis," in Proceedings of the in Proceedings of 24th International Symposium on Space Flight Dynamics (ISSFD), Laurel, Md, USA, May 2014.

[3] E. Mazarico, D. D. Rowlands, G. A. Neumann et al., "Orbit determination of the Lunar Reconnaissance Orbiter," Journal of Geodesy, vol. 86, no. 3, pp. 193-207, 2012.

[4] E. Mazarico, S. Goossens, F. G. Lemoine, G. A. Neumann, M. H. Torrence, and D. D. Rowlands, "Improved orbit determination of lunar orbiters with lunar gravity fields obtained by the GRAIL mission," in Proceedings of 44th Lunar and Planetary Science Conference, LPI Contribution No. 1719, Woodlands, Tex, USA, March 2013.

[5] J. Yan, Z. Zhong, F. Li et al., "Comparison analyses on the $150 \times 150$ lunar gravity field models by gravity/topography admittance, correlation and precision orbit determination," Advances in Space Research, vol. 52, no. 3, pp. 512-520, 2013.

[6] A. Maier and O. Baur, "Orbit determination and gravity field recovery from Doppler tracking data to the Lunar Reconnaissance Orbiter," Planetary and Space Science, vol. 122, pp. 94-100, 2016.

[7] F. G. R. Lemoine, D. E. Smith, M. T. Zuber, G. A. Neumann, and D. D. Rowlands, "A 70th degree lunar gravity model (GLGM-2) from Clementine and other tracking data," Journal of Geophysical Research: Planets, vol. 102, no. 7, Article ID 97JE01418, pp. 16339-16359, 1997.

[8] A. S. Konopliv and D. N. Yuan, "Lunar Prospector 100th degree gravity model development," in in Proceedings of 30th Lunar and Planetary Science Conference 1067, vol. 281, Houston, Tex, USA, March 1999.
[9] A. S. Konopliv, S. W. Asmar, E. Carranza, W. L. Sjogren, and D. N. Yuan, "Recent gravity models as a result of the Lunar Prospector mission," Icarus, vol. 150, no. 1, pp. 1-8, 2001.

[10] M. T. Zuber, D. E. Smith, M. M. Watkins et al., "Gravity field of the moon from the Gravity Recovery and Interior Laboratory (GRAIL) mission," Science, vol. 339, no. 6120, pp. 668-671, 2013.

[11] A. S. Konopliv, R. S. Park, D.-N. Yuan et al., "The JPL lunar gravity field to spherical harmonic degree 660 from the GRAIL Primary Mission," Journal of Geophysical Research: Planets, vol. 118, no. 7, pp. 1415-1434, 2013.

[12] A. S. Konopliv, R. S. Park, D.-N. Yuan et al., "High-resolution lunar gravity fields from the GRAIL Primary and Extended Missions," Geophysical Research Letters, vol. 41, no. 5, pp. 14521458, 2014.

[13] F. G. Lemoine, S. Goossens, T. J. Sabaka et al., "GRGM900C: A degree 900 lunar gravity model from GRAIL primary and extended mission data," Geophysical Research Letters, vol. 41, no. 10, pp. 3382-3389, 2014.

[14] D. Arnold, S. Bertone, A. Jäggi, G. Beutler, and L. Mervart, "GRAIL gravity field determination using the Celestial Mechanics Approach," Icarus, vol. 261, pp. 182-192, 2015.

[15] S. Krauss, B. Klinger, O. Baur, and T. Mayer-Gürr, "Development of the lunar gravity field model GrazLGM300a," Vermessung Geoinformation 2+3, pp. 156-161, 2015.

[16] D. A. Vallado, R. S. Hujsak, T. M. Johnson, J. H. Seago, and J. W. Woodburn, "Orbit determination using ODTK version 6," in Proceedings of the in Proceedings of 4th International Conference on Astrodynamics Tools and Techniques (ICATT), Madrid, Spain, May 2010.

[17] L. Policastri, J. P. Carrico, C. Nickel, A. Kam, R. Lebois, and R. Sherman, "Orbit determination and acquisition for LADEE and LLCD mission operations," in Proceedings of the in Proceedings of 25th AAS/AIAA Space Flight Mechanics Meeting, Williamsburg, Va, USA, January 2015.

[18] S. Slojkowski, J. Lowe, and J. Woodburn, "Orbit determination for the Lunar Reconnaissance Orbiter using an extended Kalman filter," in Proceedings of the in Proceedings of 25th International Symposium on Space Flight Dynamics (ISSFD), Munich, Germany, October 2015.

[19] E. Fantino and S. Casotto, "Methods of harmonic synthesis for global geopotential models and their first-, second- and thirdorder gradients," Journal of Geodesy, vol. 83, no. 7, pp. 595-619, 2009. 


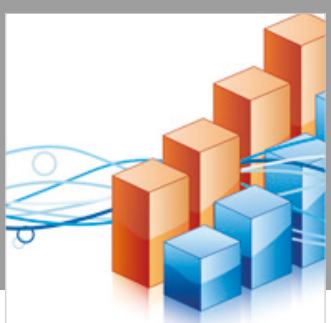

Advances in

Operations Research

\section{-n-m}
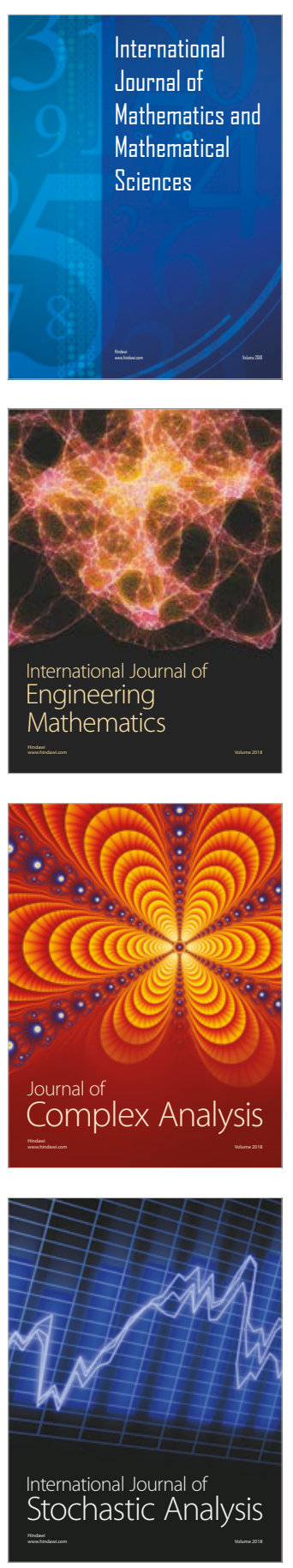
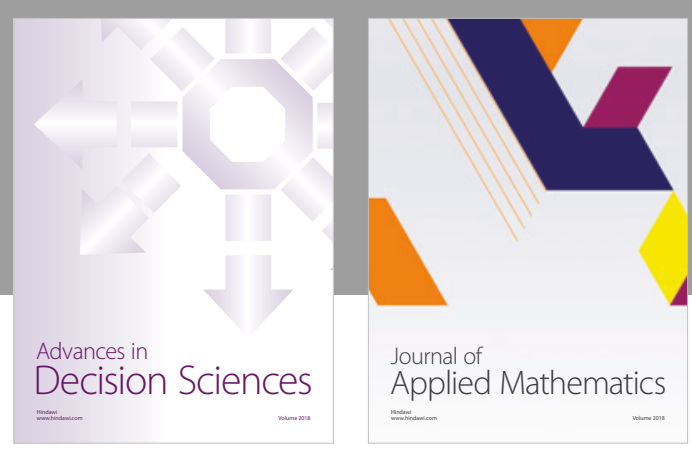

Journal of

Applied Mathematics
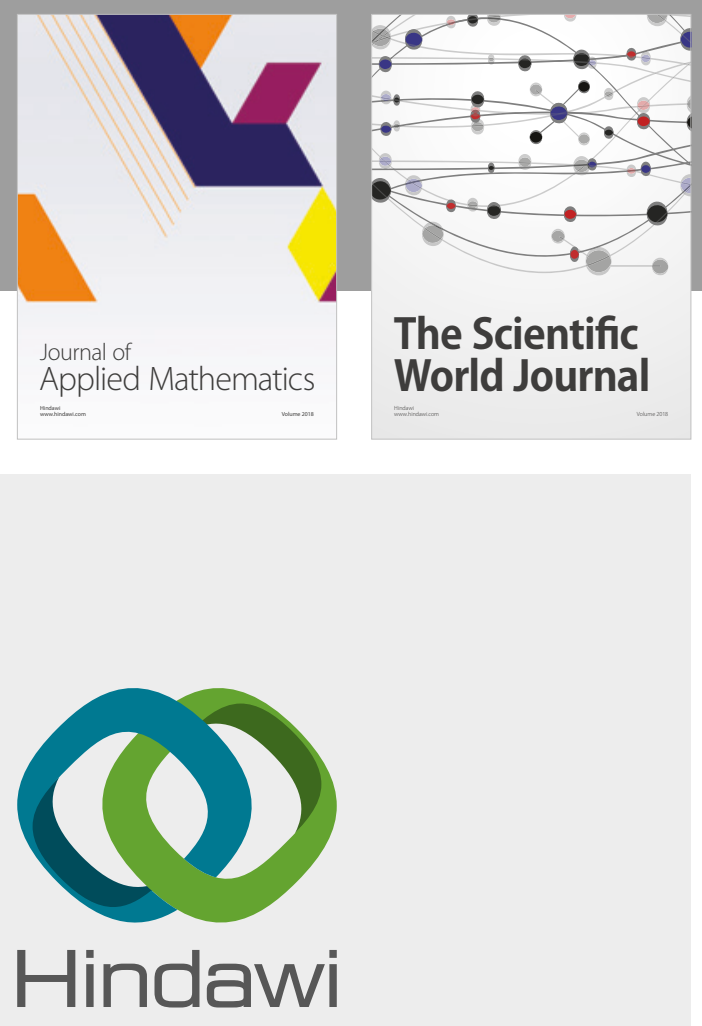

Submit your manuscripts at

www.hindawi.com

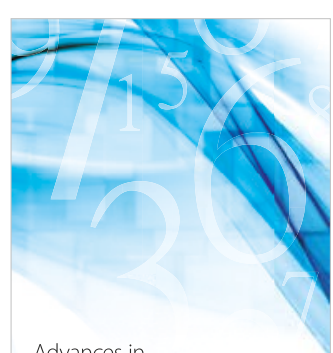

Advances in
Numerical Analysis
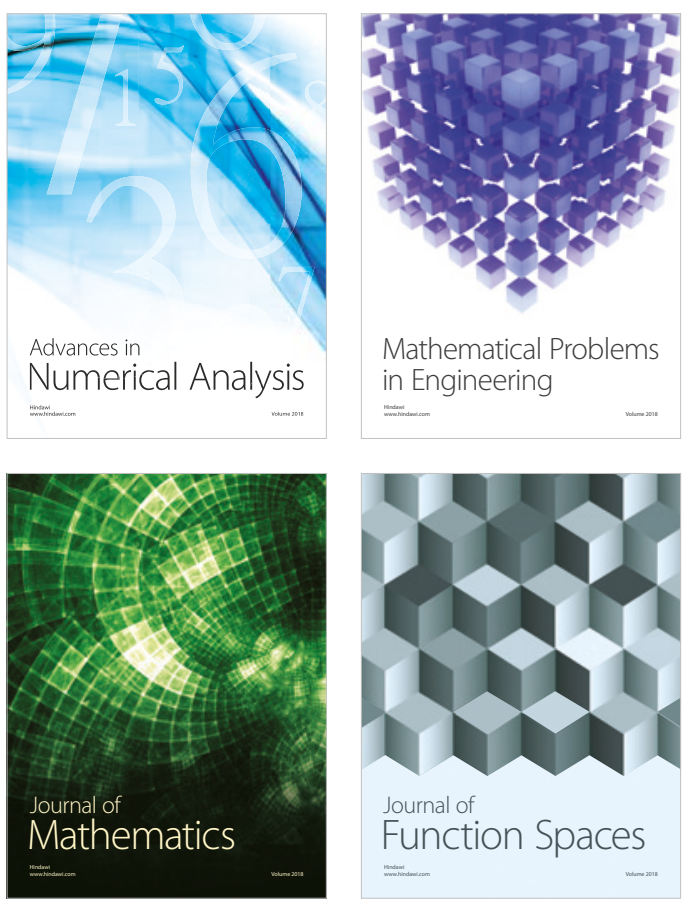

Mathematical Problems in Engineering

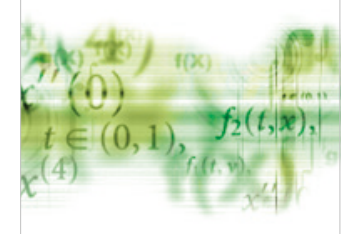

International Journal of

Differential Equations

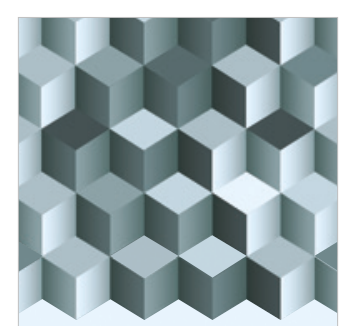

Journal of

Function Spaces

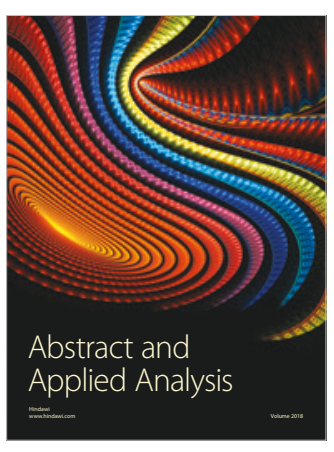

The Scientific

World Journal

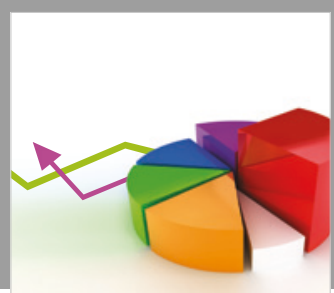

Journal of

Probability and Statistics
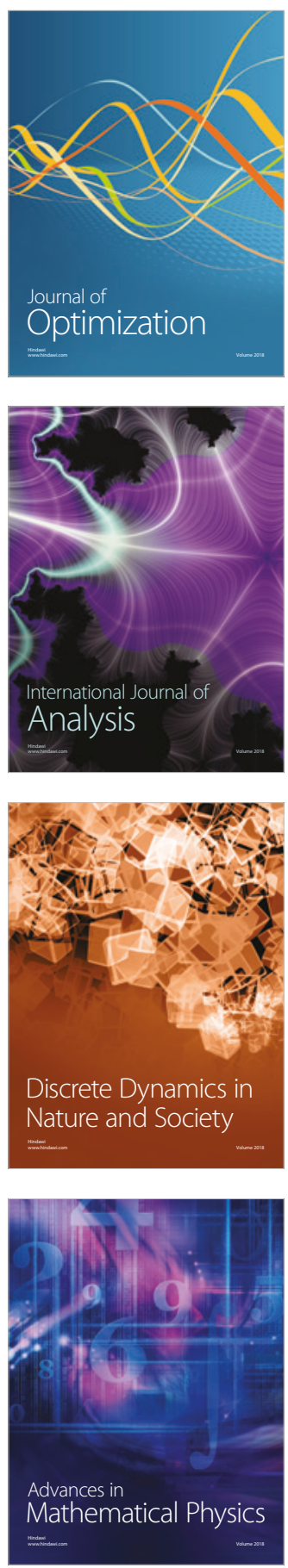